\title{
Axonal Regrowth after Spinal Cord Injury via Chondroitinase and the Tissue Plasminogen Activator (tPA)/Plasmin System
}

\author{
Noreen Bukhari, ${ }^{1,3}$ Luisa Torres, ${ }^{2,3}$ John K. Robinson, ${ }^{4}$ and Stella E. Tsirka ${ }^{1,2,3}$ \\ ${ }^{1}$ Program in Neuroscience, ${ }^{2}$ Program in Molecular and Cellular Pharmacology, and ${ }^{3}$ Department of Pharmacological Sciences, Stony Brook University, New \\ York 11794-8651, and 4Biological Psychology, Department of Psychology, Stony Brook University, Stony Brook, New York 11794-2500
}

Spinal cord injury (SCI) causes permanent debilitation due to the inability of axons to grow through established scars. Both the sugar chains and core proteins of chondroitin sulfate proteoglycans (CSPGs) are inhibitory for neurite regrowth. Chondroitinase $\mathrm{ABC}$ (ChABC) degrades the sugar chains and allows for synaptic plasticity, suggesting that after the sugar chain cleavage additional steps occur promoting a permissive microenvironment in the glial scar region. We report that the clearance of the core protein by the tissue plasminogen activator (tPA)/plasmin proteolytic system partially contributes to ChABC-promoted plasticity. tPA and plasmin are upregulated after SCI and degrade the deglycosylated CSPG proteins. Mice lacking tPA (tPA ${ }^{-/-}$) exhibit attenuated neurite outgrowth and blunted sensory and motor recovery despite $\mathrm{ChABC}$ treatment. Coadministration of $\mathrm{ChABC}$ and plasmin enhanced the $\mathrm{PAA}^{-1-}$ pheno- $^{-}$ type and supported recovery in WT SCI mice. Collectively, these findings show that the tPA/plasmin cascade may act downstream of ChABC to allow for synergistic sensory and motor improvement compared with each treatment alone and suggest a potential new approach to enhance functional recovery after SCI.

\section{Introduction}

CNS axons fail to regenerate after spinal cord injury (SCI), partially due to the accumulation of extracellular matrix (ECM) molecules in the lesion and formation of the glial scar. Among the ECM are CSPGs whose deposition is associated with inhibition of axon regrowth (McKeon et al., 1991; Smith-Thomas et al., 1994; Davies et al., 1997; Niederost et al., 1999). Chondroitin sulfate proteoglycans (CSPGs) contain a protein core covalently linked to glycosaminoglycan (GAG) chains (Morgenstern et al., 2002). Neurocan, brevican, versican, and aggrecan are lecticans (Jones et al., 2003); NG2 and phosphacan are unique. NG2 exists either as transmembrane protein, or is shed and deposited (Nishiyama et al., 1991; Jones et al., 2002). Phosphacan corresponds to the extracellular domain of receptor protein tyrosine phosphatase $\beta$ (Yick et al., 2000; Moon et al., 2001; Bradbury et al., 2002; Fulmer, 2009).

Chondroitinase $\mathrm{ABC}$ (ChABC) deglycosylates CSPGs removing GAG chains. Deglycosylation results in enhancement of synaptic plasticity to varying degrees after SCI (Busch et al., 2010;

Received July 1, 2011; revised Aug. 23, 2011; accepted Aug. 25, 2011.

Author contributions: N.B., J.K.R., and S.E.T. designed research;N.B. and L.T. performed research; N.B., J.K.R., and S.E.T. analyzed data; N.B. and S.E.T. wrote the paper.

This work was funded by NIH Grant F30 NS064676 NRSA (to N.B.), and by NIH Grant R01NS42168 and New York State Department of Health Grant SCRIB44 (to S.E.T.). We thank Drs. Michael Frohman, Lorne Mendell, and Joel Levine for critical reading of this manuscript, Dr. Joel Levine for NG2 protein and antibody, and Tsirka lab members and the Stony Brook University Medical Scientist Training Program for helpful suggestions.

Correspondence should be addressed to Dr. Stella Tsirka, Department of Pharmacological Sciences, BST8-192, Stony Brook University, New York, NY 11794-8651. E-mail: stella@pharm.stonybrook.edu.

DOI:10.1523/JNEUROSCI.3339-11.2011

Copyright $\odot 2011$ the authors $\quad 0270-6474 / 11 / 3114931-13 \$ 15.00 / 0$
Hellal et al., 2011) via an unknown mechanism. In vitro cleaved GAG chains serve as neuronal guidance cues and inhibit axonal growth (Laabs et al., 2007; Wang et al., 2008). In culture (Oohira et al., 1991; Dou and Levine, 1994) CSPG core proteins inhibit neurite outgrowth. The core protein effect has not been assessed in vivo.

Tissue plasminogen activator tPA is a serine protease that mediates proteolytic conversion of plasminogen to plasmin resulting in blood clot resolution (Collen, 1999). In the CNS tPA is secreted by neurons and microglia, activating the latter through a nonproteolytic pathway (Tsirka et al., 1995, 1997). Studies exploring the role of tPA in SCI suggest that proteolytic and nonproteolytic mechanisms are active. Nonproteolytic tPA recruits microglia which then interact with myelin basic protein and result in axonal demyelination after SCI (Abe et al., 2003). Proteolytic pathways in SCI have shown that both tPA and urokinase plasminogen activator (uPA) participate to recover respiratory function (Minor and Seeds, 2008; Seeds et al., 2009). tPA's substrate, plasminogen, and product, plasmin, can degrade phosphacan in an epilepsy model (Wu et al., 2000) and NG2 in an ex vivo SCI model (Nolin et al., 2008), and in decorin-mediated degradation of neurocan and brevican in rat SCI (Davies et al., 2004, 2006).

After seizures tPA/plasmin degrade neurocan and phosphacan in the brain and promote neurite reorganization (Wu et al., 2000). In in vitro and ex vivo settings GAG removal from NG2 enhances its interaction with $\mathrm{tPA} /$ plasmin(ogen), suggesting that CSPG core proteins may function as scaffold for tPA binding and efficient generation of plasmin. Plasmin then degrades the CSPG core (Nolin et al., 2008). We assessed the contribution of tPA/ plasmin to ChABC-promoted SCI recovery hypothesizing that 
when ChABC cleaves GAGs, it both removes an inhibitory CSPG component and allows tPA/plasmin to clear the core protein. Although individually tPA/plasmin and ChABC can promote synaptic plasticity and motor improvements, we think that synergistic interaction of the proteases and ChABC on CSPGs in the glial scar may allow for greater neurite regrowth and synaptic plasticity after SCI.

\section{Materials and Methods}

Animals and surgery

All experiments conform to the NIH guidelines and were approved by the Department of Laboratory Animal Research at Stony Brook University. tPA knock-out (KO) mice have been backcrossed for 12 generations to the C57BL/6 background. Age and gender-matched adult C57BL/6 (WT) and tPA KO mice weighing 25-30 g were anesthetized with isoflurane and placed in a stereotaxic apparatus. A dorsal laminectomy was performed between thoracic levels 8 and 10 and spinal cord stabilized with fine forceps. Animals were then transferred to an Infinite Horizon Impactor (Precision Systems and Instrumentation) and a $50 \mathrm{kdyn}$ impactor tip with $1.25 \mathrm{~mm}$ tip diameter was dropped from a height of $2 \mathrm{~cm}$ on the central canal between T8 and T10. The overlying muscle and skin were sutured. Sham-operated groups underwent laminectomy without contusion. Postoperatively, mice were injected with buprenorphine $(0.03 \mathrm{mg} / \mathrm{kg})$ subcutaneously to reduce pain and placed on a heating pad for $24 \mathrm{~h}$ to recover. Mice were then transferred to $27^{\circ} \mathrm{C}$ temperature controlled room, and food pellets and liquid solution (Napa) were placed at the bottom of their cages. Daily weight measurements were performed. Bladders were expressed twice daily, and $0.6-0.8 \mathrm{cc}$ of $5 \%$ dextrose/saline injected subcutaneously for underweight animals $(<25 \mathrm{~g})$ until end of experiment.

\section{Tissue processing}

Spinal cord tissue was processed in one of two ways. To prepare spinal cord homogenates, mice were terminally anesthetized with $2.5 \%$ avertin and transcardially perfused with PBS. Using the injury epicenter as the origin, $2 \mathrm{~mm}$ each in the rostral and caudal direction of the spinal cord were isolated and suspended in Tris-buffer saline, $\mathrm{pH}$ 7.0. Isolated spinal cord was manually homogenized and centrifuged at $20,800 \times g$ for 30 min at $4^{\circ} \mathrm{C}$. Supernatant was collected and total protein content measured using the Bio-Rad Bradford protein assay. To prepare spinal cord sections, mice were transcardially perfused and postfixed with $4 \%$ PFA, $\mathrm{pH} 7.4$, cryoprotected with $30 \%$ sucrose overnight at $4^{\circ} \mathrm{C}$, and frozen in OCT (Triangle Biomedical Sciences). Sagittal sections $(18 \mu \mathrm{m})$ through the thoracic spinal cord were prepared. All spinal cord samples were stored at $-80^{\circ} \mathrm{C}$ until further use.

\section{Zymography}

Fourteen day SCI homogenates and embryonic day 14 cortical neurons from WT and tPA KO mice ( $20 \mu \mathrm{g}$ of protein/sample) were analyzed on $10 \%$ SDS-PAGE copolymerized with plasminogen $(13 \mathrm{mg} / \mathrm{ml})$ and casein $(3 \mathrm{mg} / \mathrm{ml})$. After electrophoresis, SDS was removed by incubating the gel with $2.5 \%$ TX-100 for an hour. The gel was then incubated with $0.1 \mathrm{M}$ Tris, $\mathrm{pH} 8.0$, overnight at $37^{\circ} \mathrm{C}$. The next day, gel was stained with Coomassie blue for $2 \mathrm{~h}$ and destained until clear zones of lysis became visible. Pure, recombinant tPA (500 ng; Genentech) was used as positive control.

\section{Amidolytic assay}

SCI homogenates and cortical neuron cell lysates and media from WT and tPA KO mice were incubated with $0.68 \mu \mathrm{M}$ plasminogen in a $50 \mathrm{~mm}$ Tris buffer, $\mathrm{pH} 8.1$, with $2 \% \mathrm{BSA}$ and $0.3 \mathrm{~mm}$ chromogenic substrate2251 (DiaPharma) overnight in the dark at room temperature. Cleavage of the chromogenic substrate S-2251 by serine protease-generated plasmin and the subsequent color change was quantified at $405 \mathrm{~nm}$. A doseresponse for pure recombinant tPA activity was simultaneously run alongside the test samples and the time point when the $R^{2}$ value for the dose-response was closest to 1 was used to measure the concentration of serine protease in the samples. All samples were run in triplicates. Amiloride (1 mM) was used to block uPA in the indicated samples.

\section{Western blot}

WT and tPA KO sham and SCI homogenates $(50 \mu \mathrm{g})$ were treated with ChABC $(0.5 \mathrm{U} / \mathrm{ml}$; Seikagaku $)$ in $100 \mathrm{~mm}$ Tris-acetate, $\mathrm{pH} 7.4$, for $3 \mathrm{~h}$ at $37^{\circ} \mathrm{C}$ on coverslips. Sample buffer containing $5 \% \beta$-mercaptoethanol was then added and the protein mix denatured at $100^{\circ} \mathrm{C}$ for $10 \mathrm{~min}$. Samples were run on $6 \%$ SDS-PAGE containing Tris-glycine and transferred onto polyvinylidene fluoride membrane. Membranes were blocked in 5\% milk in PBS for $1 \mathrm{~h}$ and probed overnight with one of the following primary antibodies: NG2 (1:500, rabbit anti-NG2; gift from Joel Levine, Stony Brook University, Stony Brook, NY), Neurocan [1: 100; mouse-1F6; Developmental Studies Hybridoma Bank (DSHB)], plasminogen (1:1000; anti-mouse plasminogen; Millipore), GFAP (1: 1000; anti-rabbit; Abcam), Iba-1 (1:1000; anti-rabbit; WAKO). The following day, membranes were washed with PBS-T and incubated with the species-appropriate peroxidase-conjugated secondary antibody (1: 2000-1:/5000; Vector Labs) for $1 \mathrm{~h}$ at room temperature. After multiple washes with PBS-T, antibody binding was visualized using chemiluminescence kit according to the manufacturer's protocol (Thermo Scientific). For pure protein experiments, $10 \mathrm{ng}$ of pure NG2 (gift from Dr. Joel Levine) or $2.5 \mu \mathrm{g}$ of CSPG mix (CC117; Millipore Bioscience Research Reagents) were incubated with plasminogen $(0.68 \mu \mathrm{M})$ and pure recombinant $\mathrm{tPA}$ or $\mathrm{uPA}(50,100$, and $500 \mathrm{U} / \mu \mathrm{g})$ with or without $\mathrm{ChABC}$ and Tris-acetate buffer as above. To confirm equal loading, membranes were stripped with $0.2 \mathrm{M}$ Tris-Glycine, $\mathrm{pH} 2.2$, and $0.5 \mathrm{~m}$ $\mathrm{NaCl}$ strip buffer overnight at room temperature. Samples were reprobed with $\alpha$-tubulin antibody (1:2000; Sigma). Protein bands were quantified by densitometry in Image (NIH) software and data normalized against $\alpha$-tubulin protein bands.

\section{Neurite outgrowth assay}

Twenty-five millimeter coverslips were UV-irradiated and coated overnight with poly-D-lysine $(25 \mu \mathrm{g} / \mathrm{ml})$ and laminin $(10 \mu \mathrm{g} / \mathrm{ml})$ at $37^{\circ} \mathrm{C}$. The next day, embryonic day 15 cortices were isolated from WT and tPA KO mice and manually dissociated in Neurobasal Media with B27 supplement (Invitrogen), L-glutamine, gentamicin, and Penicillin/streptomycin. Cells were plated at a density of 100,000 cells/coverslip on WT or tPA KO Sham and SCI homogenates previously treated with ChABC or penicillinase (Pen; $0.5 \mathrm{U} / \mathrm{ml}$ ) in vitro for $3 \mathrm{~h}$ at $37^{\circ} \mathrm{C}$ as detailed in Western blot procedure. After $2 \mathrm{~d}$ in culture, cells were fixed with $4 \%$ PFA for $1 \mathrm{~h}$, blocked with $2 \%$ goat serum and incubated overnight with CSPG (1:200 CS56; Sigma). The next day, cells were washed in PBS and incubated with goat anti-mouse-Alexa 555 secondary antibody (1:1000; Invitrogen) for $1 \mathrm{~h}$ followed by a second overnight incubation with $\beta$-tubulin III antibody (1:2000; Covance). On the last day, cells were washed again in PBS and incubated with goat anti-rabbit Alexa 488 antibody (1:2000; Invitrogen) and coverslipped with mounting media (Fluoromount).

Images were captured with a Zeiss confocal microscope using LSM 510 Meta software. A grid format was used with CSPG-rich regions in each grid first identified and images of neurons on the CSPG-rich region then captured. Twenty-five different regions were sampled per biological replicate. Data were calculated as a ratio of the total neurite length/neuron for each biological replicate. Total neurite length in each image panel was quantified using NeuriteTracer plug-in in ImageJ (Pool et al., 2008) while total neurons were manually counted in each image panel. Data were normalized against cortical neurons only images of the same genotype and plotted as a percentage of neurite length/neuron. Approximately 400-600 neurons were quantified per biological replicate.

\section{In vivo $C h A B C$ treatment}

Two weeks after contusion injury, WT and tPA KO mice were reanesthetized and placed in a stereotaxic apparatus. The injured region was reexposed and $1 \mu \mathrm{l}$ of ChABC (50 U/ml, Seikagaku) or Pen $(50 \mathrm{U} / \mathrm{ml}$, Sigma catalog \# P0389, $1 \mathrm{kU}$,) was injected into the central canal (1 mm depth, rate of $0.4 \mu \mathrm{l} / \mathrm{min}$ ) using a 28 -gauge Hamilton syringe attached to an automated microinjector. The needle was withdrawn after an additional 1 min to prevent reflux.

\section{In vivo immunohistochemistry}

Two days after ChABC injection, mice were terminally anesthetized, fixed with $4 \%$ PFA and sagittal spinal cord sections prepared as previ- 
ously outlined. Sections were sequentially blocked with mouse blocking serum (Vector Labs) and goat serum, and then briefly incubated in mouse diluent before probing with one of the following primary antibodies for $30 \mathrm{~min}$ at room temperature: CSPG (1:200, CS56; Sigma), NG2 (1:1000, rabbit anti-NG2), Neurocan (1:50, anti-mouse 1F6; DSHB), Phosphacan (1:100, mouse-3F8; DSHB), or chondroitin-4sulfate (C4S, mouse-MAB2030, 1:1000; Millipore Bioscience Research Reagents). Species-appropriate Alexa 488 antibody was then applied followed by overnight incubation with glial fibrillary acidic protein (GFAP 1:500; DAKO) to outline the glial scar. Species-appropriate-Alexa 555 antibody was then applied and sections were mounted with the nuclear labeling mounting medium. To quantify, multiple images around the glial scar border (indicated by upregulated GFAP expression) of each section were captured at a digital resolution of $1024 \times 1024$ with a Zeiss confocal microscope using LSM 510 Meta software. A sagittal section every 10 sections $(180 \mu \mathrm{m})$ apart was imaged per biological replicate. Image (NIH) was used to quantify the total mean intensity/area for each biological replicate and each antibody staining. Representative images were oriented with dorsal side up in Adobe Photoshop 7.0.1.

\section{Motor behavior}

Rotarod. Intact and injured WT and tPA $\mathrm{KO}$ mice were placed on a standard Rotarod (Med Associates) to assess motor performance. The rod rotation accelerated over the course of the $5 \mathrm{~min}$ from 4 to 40 rotations per minute. Each mouse was given three trials to remain on the accelerating rod per day. Each trial was 5 min long or until the mouse fell off. The maximum speed and latency to fall was recorded automatically by the apparatus. The maximum speed each mouse remained on the rotor rod in the three trials per day was used for comparisons.

Open field activity. Intact and injured WT and $\mathrm{PAA} \mathrm{KO}$ mice were placed in an empty rat cage $(44 \mathrm{~cm} \times 21 \mathrm{~cm})$ situated inside an OptoVarimex-Minor animal activity meter (Columbus Instruments). Infrared beams $(15 \times 15)$ running in the $x-y$ coordinates and $2 \mathrm{~cm}$ above the base of the machine recorded beam breaks associated with ambulatory activity (consecutive beam breaks) and total activity (all beam interruptions) during the $20 \mathrm{~min}$ observation period. An observer, blind to genotype also recorded the animal's supported motor movements. Supported motor movements or full rearings were defined as an animal using its upper and lower limbs to reach up the side of a cage and hold its body up (supported) or stand up on its lower limbs without using any cage support (unsupported). Unsupported movements were infrequent in all groups, so not presented. Behavior was recorded 7, 10, 18, and $21 \mathrm{~d}$ after contusion injury.

\section{Sensory axon tracing}

On day 22, mice were reanesthetized and the right sciatic nerve exposed. Cholera toxin B subunit (CTB, $2.5 \mu \mathrm{l}, 1.5 \%$ ) (List Biological Laboratories) was injected into the nerve with a 32 gauge Hamilton syringe connected to an automated microinjector $(0.4 \mu \mathrm{l} / \mathrm{min})$. The needle was withdrawn after an additional minute to prevent reflux. Overlying muscle and skin was sutured and mice were left on heating pad to recover. Spinal cords were isolated $4 \mathrm{~d}$ later.

To analyze axonal regrowth, 4 serial sagittal sections were labeled at an interval of 10 sections with goat anti-CTB antibody (1:5000; List Biological Laboratories) followed by a second probe with biotinylated rabbit anti-goat IgG (Vector labs). Sections were then sequentially incubated with avidin-biotinylated horse-radish peroxidase and 3,3' -diaminobenzidine tetrahydrochloride (DAB) to visualize the axons. Silver-gold reaction was used to intensify axon staining. To measure axon density, Stereoinvestigator (MicroBrightField) software was used to outline the injured glial scar region, $800 \mu \mathrm{m}$ rostral and $800 \mu \mathrm{m}$ caudal to the injury epicenter for each of the four sections in every spinal cord. The software then created a grid format within the outlined glial scar, rostral and caudal regions to sequentially and randomly sample multiple areas within each region. Axons found within each region were marked by an observer. The software then measured the total axonal length of all the marked axons to provide values of total axonal length in each outlined region (glial scar, rostral and caudal) of the spinal cord. Data were then exported to Microsoft Excel and the total regional axonal length was divided by the regional tissue volume to calculate axonal density/spinal cord region $\left(\mu \mathrm{m} / \mu \mathrm{m}^{3}\right)$. Data were normalized against axonal density of the caudal region of every spinal cord and plotted as a percentage of axon density in caudal region (Tan et al., 2006, 2007).

\section{Statistics}

For behavior and axon tracing experiments, all surgeries and measurements were performed with observers blinded to the genotype/treatment of the mice. To compare the two genotypes within each treatment or time point, $t$ test was used. Tests used for multiple group comparisons are presented in the figure legends. Significant multiple group comparisons were followed by post hoc Holm-Sidak test in all cases. A minimum $\alpha$-value of 0.05 was accepted as statistically significant throughout. Data are presented as mean \pm SEM.

\section{Results}

\section{Severity and variability of contusion injury model}

To evaluate the severity and variability of the contusion injury model, we compared the survival of sham and contusion-injured mice across genotypes and treatments in each experiment (Table 1). A total of 213 mice underwent sham or contusion injury with a 15\% mortality rate [15 WT and 17 mice lacking tPA (tPA KO)]. The highest mortality was seen in the behavioral experiments ( 7 WT and 10 tPA KO died). To assess for any additional differences in contusion injury between the genotypes, we also measured the body weight throughout the behavioral experiment (Fig. 1). No significant weight differences were measured between the WT and tPA KO contusion-injured mice compared with their uninjured weights (Fig. 1a,b). Similarly no significant weight differences due to treatment (Fig. $1 c, d$; treatment indicated by dashed line) or genotype and treatment on the last day of the experiment (Fig. 1e) were noted. Therefore, although our contusion injury model shows a moderate injury severity in general, the variability in severity of the injury between genotypes or treatments in the experiments was not statistically significant.

\section{Upregulation of tPA and $\mathrm{uPA}$ activity after contusion injury}

We previously reported an interaction between ChABC, the tPA/ plasmin cascade, and the CSPG molecule NG2 using biochemical techniques, and ex vivo techniques after SCI (Nolin et al., 2008). Here we explored the interactions between PA/plasmin and CSPGs in the in vivo setting of SCI. Sham and injured spinal cords from WT and tPA KO mice were isolated at $4 \mathrm{~h}$, and at 3, 7, and $14 \mathrm{~d}$ after contusion injury, and tissue was isolated $2 \mathrm{~mm}$ rostrally and caudally from injury center. The tissue was homogenized and used to measure serine protease activity. Serine protease activity was significantly upregulated within $4 \mathrm{~h}$ of SCI in WT mice and remained elevated for at least $14 \mathrm{~d}$ (Fig. 2a). Increases in serine protease activity were also seen in SCI IPA KO mice, although the levels of activity were much lower in both the sham and experimental groups. The urokinase blocker (Vassalli and Belin, 1987), amiloride, was used to efficiently inhibit uPA (Fig. 2b) as a means to specifically measure tPA activity; upregulation of specific tPA activity was then observed in WT SCI mice, but not in tPA KO SCI mice as demonstrated by others (Veeravalli et al., 2009) (Fig. $2 b)$. Zymography confirmed the presence of tPA $(68 \mathrm{kDa})$ and $\mathrm{uPA}(33 \mathrm{kDa})$ activities in WT mice $14 \mathrm{~d}$ after contusion injury, but only uPA in tPA KO SCI mice (Fig. 2c). Thus, both tPA and uPA activities are present and upregulated after SCI, and based on these findings, day 14 after SCI was used for analysis in subsequent experiments.

\section{tPA/plasmin system specifically degrades NG2 and phosphacan after ChABC cleavage in vivo}

To examine whether ChABC-treated CSPG proteins become substrates for the $\mathrm{tPA} /$ plasmin system, we performed contusion 
Table 1. An overview of sham and $\mathrm{SCl}$ animals used, categorized by experiment, genotype, and treatment

\begin{tabular}{|c|c|c|c|c|c|c|}
\hline Experiment & Genotype & Injury & Treatment & Number of mice & Exp endpoint (days) & Died-(days post-SCI) \\
\hline \multirow[t]{4}{*}{ Amidolytic } & \multirow[t]{2}{*}{ WT } & Sham & - & 3 & 14 & 0 \\
\hline & & Contusion & - & 12 & $4 \mathrm{~h}, 3,7,14$ & 0 \\
\hline & \multirow[t]{2}{*}{ tPA KO } & Sham & - & 3 & 14 & 0 \\
\hline & & Contusion & - & 12 & $4 h, 3,7,14$ & 0 \\
\hline \multirow[t]{4}{*}{ Zymography } & \multirow[t]{2}{*}{ WT } & Sham & - & 4 & 14 & 0 \\
\hline & & Contusion & - & 5 & 14 & $1-(5)$ \\
\hline & \multirow[t]{2}{*}{ tPA KO } & Sham & - & 4 & 14 & 0 \\
\hline & & Contusion & - & 5 & 14 & $1-(2)$ \\
\hline \multirow[t]{6}{*}{$\mathrm{IHC}$} & \multirow[t]{3}{*}{ WT } & Sham & - & 3 & 16 & 0 \\
\hline & & Contusion & Pen & 4 & 16 & $1-(14)$ \\
\hline & & Contusion & ChABC & 4 & 16 & 0 \\
\hline & \multirow[t]{3}{*}{ tPA KO } & Sham & - & 3 & 16 & 0 \\
\hline & & Contusion & Pen & 4 & 16 & 0 \\
\hline & & Contusion & $\mathrm{ChABC}$ & 4 & 16 & $1(5)$ \\
\hline \multirow[t]{4}{*}{ WB and neurite assay homogenate } & \multirow[t]{2}{*}{ WT } & Sham & - & 3 & 14 & 0 \\
\hline & & Contusion & - & 9 & 14 & $3(4,6,8)$ \\
\hline & \multirow[t]{2}{*}{ tPA KO } & Sham & - & 3 & 14 & 0 \\
\hline & & Contusion & - & 11 & 14 & $2(0,6)$ \\
\hline \multirow[t]{8}{*}{ Behavior } & \multirow[t]{5}{*}{ WT } & Sham & - & 8 & 21 & 0 \\
\hline & & Contusion & Pen & 12 & 21 & $4(10,13,24,25)$ \\
\hline & & Contusion & Pen+Plasmin & 3 & 21 & 0 \\
\hline & & Contusion & ChABC & 15 & 21 & $3(0,2,13)$ \\
\hline & & Contusion & ChABC+ Plasmin & 5 & 21 & 0 \\
\hline & \multirow[t]{3}{*}{ tPA KO } & Sham & - & 8 & 21 & 0 \\
\hline & & Contusion & Pen & 12 & 21 & $6(2,2,3,10,12,16)$ \\
\hline & & Contusion & $\mathrm{ChABC}$ & 14 & 21 & $4(2,3,9,13)$ \\
\hline \multirow[t]{10}{*}{ Axon tracing } & \multirow[t]{5}{*}{ WT } & Sham & - & 3 & 26 & 0 \\
\hline & & Contusion & Pen & 5 & 26 & $2(10,13)$ \\
\hline & & Contusion & Pen+Plasmin & 3 & 26 & 0 \\
\hline & & Contusion & ChABC & 5 & 26 & $1(2)$ \\
\hline & & Contusion & ChABC+ Plasmin & 4 & 26 & 0 \\
\hline & \multirow[t]{5}{*}{ tPA KO } & Sham & - & 3 & 26 & 0 \\
\hline & & Contusion & Pen & 4 & 26 & $1(19)$ \\
\hline & & Contusion & Pen+Plasmin & 4 & 26 & $1(25)$ \\
\hline & & Contusion & ChABC & 5 & 26 & $1(6)$ \\
\hline & & Contusion & ChABC+ Plasmin & 4 & 26 & 0 \\
\hline
\end{tabular}

All treatments were performed on day 14 post-SCI. The numbers in parentheses in the last column denote the day of death relative to day 0 when contusion injury was performed.

IHC, Immunohistochemistry; WB, Western blot; - , no treatment.

injury on WT and tPA KO mice. Then, $14 \mathrm{~d}$ after injury, we administered ChABC $(50 \mathrm{U} / \mathrm{ml})$ or the control enzyme Pen (50 $\mathrm{U} / \mathrm{ml}$ ) intraspinally. Spinal cords were isolated $2 \mathrm{~d}$ later and CSPG deglycosylation by ChABC was assessed in the appropriate treatment groups using antibodies that reveal intact CSPGs or the remainder proteoglycans after GAG chain cleavage (Fig. 3a). The lesion area was defined using the astrocytic protein GFAP as a marker for the glial scar region (Fig. 3b). The antibody CS56 (Sigma) recognizes large oligosaccharides of CSPG GAG chains (Ito et al., 2005) and allows for visualization of the intact CSPG proteins. The immunofluorescent images show that CSPG levels are dramatically upregulated after contusion injury in the SCI+ Pen-treated groups compared with the sham group; ChABC cleavage results in significant decrease in the $\mathrm{CS}_{5}{ }^{+}$signal in glial scar regions of WT and tPA KO SCI mice (Fig. 3a,c). These results confirm previous findings that CSPGs upregulated in the glial scar region
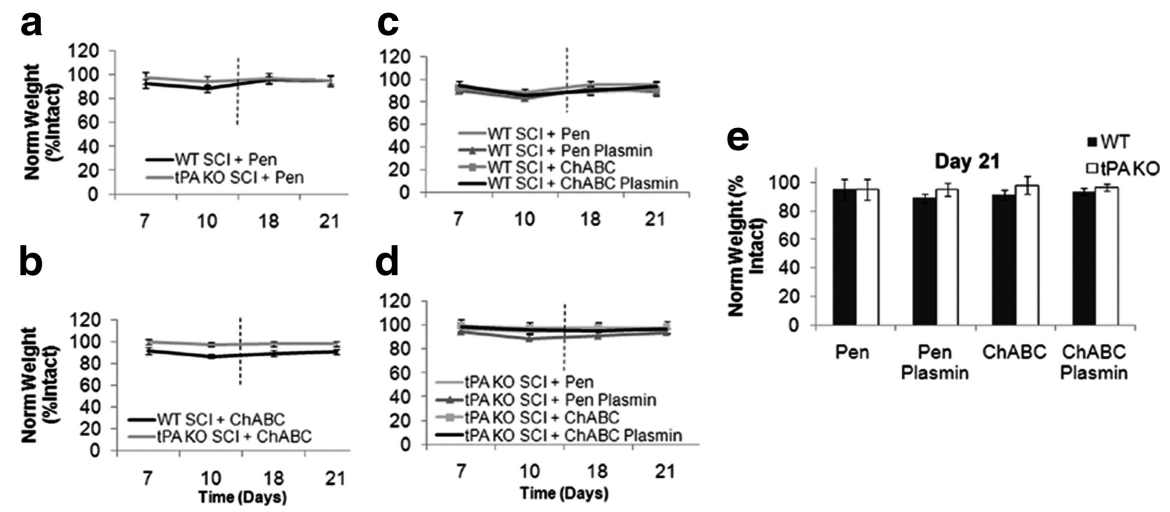

Figure 1. SCl mice retained their weight throughout the experiment. WT and $\mathrm{PA}$ KO mice underwent sham or contusion injury and a single injection of Pen, $\mathrm{ChABC}$, Pen + plasmin, or ChABC + plasmin on day 14. The weight was measured on the designated days and data were normalized to intact weight of the individual mouse of the respective genotype. Two-way group comparisons are presented in $\boldsymbol{a}-\boldsymbol{d}$. $\boldsymbol{a}$ and $\boldsymbol{b}$ compare differences due to genotype and time, while cand $\boldsymbol{d}$ illustrate differences due to treatment and time. $\boldsymbol{e}$, Weight differences due to genotype and treatment were compared on day 21. Biological replicates are indicated in Table 1 under behavioral experiment.

after contusion injury can be deglycosylated by ChABC (Moon et al., 2001; Bradbury et al., 2002).

After the GAGs are removed, the site of recognition for C4S is revealed, allowing for visualization of digested 4-sulfated CSPGs 


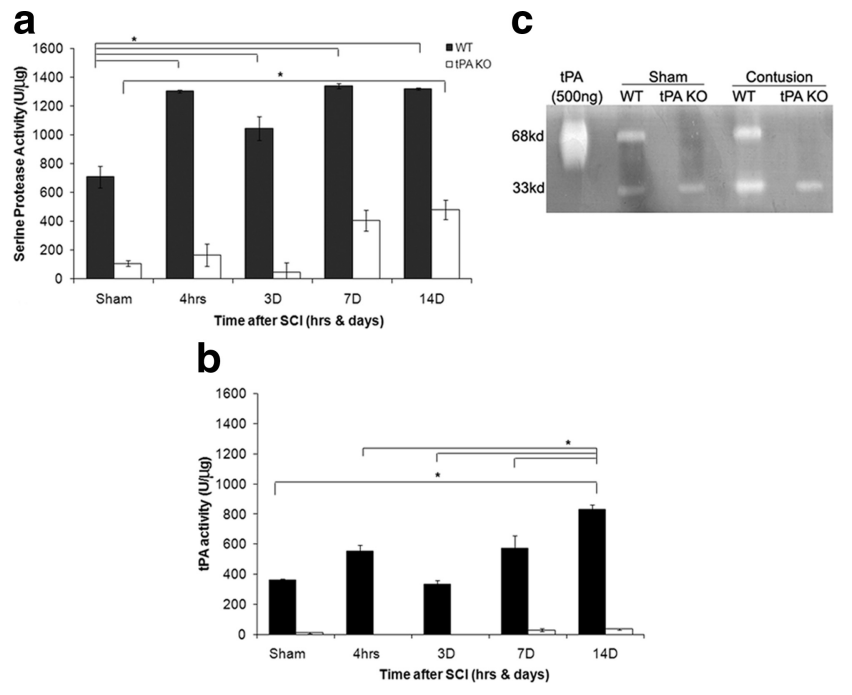

Figure 2. The tPA/plasmin(ogen) system is significantly upregulated $14 \mathrm{~d}$ after $\mathrm{SCl}$. $\boldsymbol{a}$, Serine protease activity was quantified by amidolytic assay in WT and tPA KO sham and 0 ( $4 \mathrm{~h}$ ), 3, 7, and $14 \mathrm{~d}$ after contusion injury. Repeated-measures ANOVA was used to compare across time points within each genotype $(n=3)$. Significant ANOVA values were followed by post hoc Holm-Sidak test. Significant post hoc differences are indicated by brackets and asterisks. Compared with WT sham, $p<0.001$ for serine protease activity in 0,7 , and $14 \mathrm{~d} \mathrm{SCl}$ homogenates; $p<0.05$ for serine protease activity in $3 \mathrm{~d}$ SCI homogenates. Compared with tPA KO Sham, $p<0.05$ for serine protease activity in $14 \mathrm{~d} \mathrm{SCI}$ homogenates. Amiloride, a specific uPA blocker, was used to allow the measurement of TPA activity only. $\boldsymbol{b}$, Significant repeated-measures ANOVA were followed by post hoc Holm-Sidak test $(n=3)$. Compared with WT sham, $p<0.05$ for tPA activity in $14 \mathrm{~d}$ WT SCl homogenates. c, Zymography assay was used to visualize serine protease activity in WT and TPA $K 0$ sham and $14 \mathrm{~d}$ contusion-injured mice. The $\mathrm{tPA}$ band is seen at $68 \mathrm{kDa}$ and $\mathrm{UPA}$ at $33 \mathrm{kDa}$. Pure recombinant tPA was used as positive control. The zymography assay was repeated with 4 sets of biological replicates $(n=4)$.

(Cafferty et al., 2008). C4S staining in WT SCI- and ChABCtreated mice revealed significantly lower levels of digested CSPG core proteins that localized to the border of the glial scar, while in the tPA KO animals higher core protein expression was observed that localized in the border and the epicenter of the glial scar (Fig. $3 b, d)$. These results qualitatively suggest that the absence of tPA/ plasmin system may influence the clearance of deglycosylated CSPG core proteins.

To determine the specific types of CSPG core proteins affected, we performed immunoblotting using homogenates from WT and tPA KO sham and contusion-injured mice. Protein expression could not be evaluated after the control enzyme Pen treatment using immunoblotting since the presence of GAG chains resulted in a nondistinct protein band. Instead we treated both the sham and contusion-injured groups with ChABC in vitro which resulted in significant degradation of NG2 core protein in the WT group. In the TPA KO sham group no differences were observed whereas the tPA KO contusion-injured group showed significant retention of the NG2 core protein suggesting that $\mathrm{PA}$ participated in the ChABC-mediated clearance of NG2 core protein (Fig. 4a,b). Qualitative differences in NG2 protein expression were also observed in the glial scar region of WT and tPA KO groups after treatment with the enzyme (Fig. 4d). We tested the possibility that uPA might also partially compensate for the loss of tPA by assessing in vitro the possibility that uPA can promote the degradation of the NG2 core protein by plasmin after ChABC cleavage of the GAG chains. uPA, however, was unable to use NG2 core protein as a scaffold for plasmin generation and subsequent degradation (Fig. 4h). Thus, tPA functions as the relevant serine protease in this model injury system: pre- sumably it binds to NG2 core protein and uses it as a scaffold to generate plasmin locally which will then degrade the CSPG core protein, as we have previously shown in biochemical and ex vivo experiments (Nolin et al., 2008).

We had previously reported an interaction between the tPA/ plasmin system and phosphacan and neurocan proteins after seizure in mice (Wu et al., 2000) which results in degradation of both CSPGs by plasmin. Therefore, we assessed the fate of these CSPG proteins. No statistical differences were found in the processing of neurocan core protein after ChABC cleavage in WT and tPA KO SCI homogenates using immunoblot analysis, but qualitative differences were observed with immunofluorescence (Fig. $4 a, c, e)$.In vitro cleavage experiments revealed that pure neurocan core protein could interact with both uPA and tPA (Nolin et al., 2008; Fig. $4 i$, top) and plasminogen (Fig. $4 i$, middle), and be targeted for degradation by plasmin after deglycosylation by ChABC (Fig. 4i, bottom). Thus, in the tPA KO mice, uPA may compensate by binding to neurocan core protein and targeting it for degradation by plasmin. This result is consistent with our previous reports in the spinal cord (Nolin et al., 2008) and in the brain (Wu et al., 2000), where radiolabeled core protein neurocan was processed by $\mathrm{tPA} /$ plasmin.

Although we were not able to evaluate phosphacan core protein expression using immunoblotting, immunofluorescent images and semiquantification revealed attenuated clearance of phosphacan protein after ChABC cleavage in tPA KO SCI mice compared with the corresponding WT group (Fig. $4 f, g$ ). Collectively, these results suggest that the tPA-mediated plasmin cascade may also preferentially bind and degrade phosphacan core protein.

It is possible that the removal of GAG chains from the CSPGs in the glial scar makes all other protein substrates present more accessible to proteolytic degradation. To test for this possibility, we subjected SCI protein extracts from wt and tPA KO animals that had been treated with Pen or ChABC to Western blot analysis probing for non-ChABC markers (GFAP and Iba1). Both the levels of GFAP (Fig. 4j) and Ibal (data not shown) were comparable following either Pen or $\mathrm{ChABC}$ treatment. Moreover, the presence of tPA/plasmin did not affect the GFAP or Ibal levels.

\section{The tPA/plasmin system contributes to ChABC-mediated neurite outgrowth}

ChABC cleavage of the GAG chains on CSPGs is known to enhance axon growth after SCI (Moon et al., 2001; Bradbury et al., 2002). We have previously reported that $\mathrm{tPA} /$ plasmin cleavage of NG2, phosphacan and neurocan proteins can mediate neurite outgrowth as well (Wu et al., 2000; Nolin et al., 2008). We, therefore, investigated whether the tPA/plasmin-facilitated degradation of CSPG core proteins could also affect CNS neurite outgrowth responses after ChABC cleavage. The presence of tPA activity in WT cortical neuron and its absence in TPA KO neurons was first confirmed. Active tPA was present in WT cell lysates, while the tPA KO neurons had negligible activity (Fig. $5 a, b$ ). Cortical neurons were plated on ex vivo glial scars treated with Pen or ChABC in vitro and neurite outgrowth assessed. To assess first whether the SCI homogenates were susceptible to proteolytic degradation during the treatment of the ex vivo glial scar, we incubated the SCI extracts with or without protease inhibitor cocktail (PIC) ( $1 \times$; Sigma), and/or ChABC $(0.5 \mathrm{U} / \mathrm{ml})$ for $3 \mathrm{~h}$ at $37^{\circ} \mathrm{C}$ and evaluated the integrity of neurocan protein No significant difference in neurocan levels was observed in the presence or absence of PIC (Fig. 5c). When we studied the neurite outgrowth on this substrate, we observed that cortical neurons grew exten- 

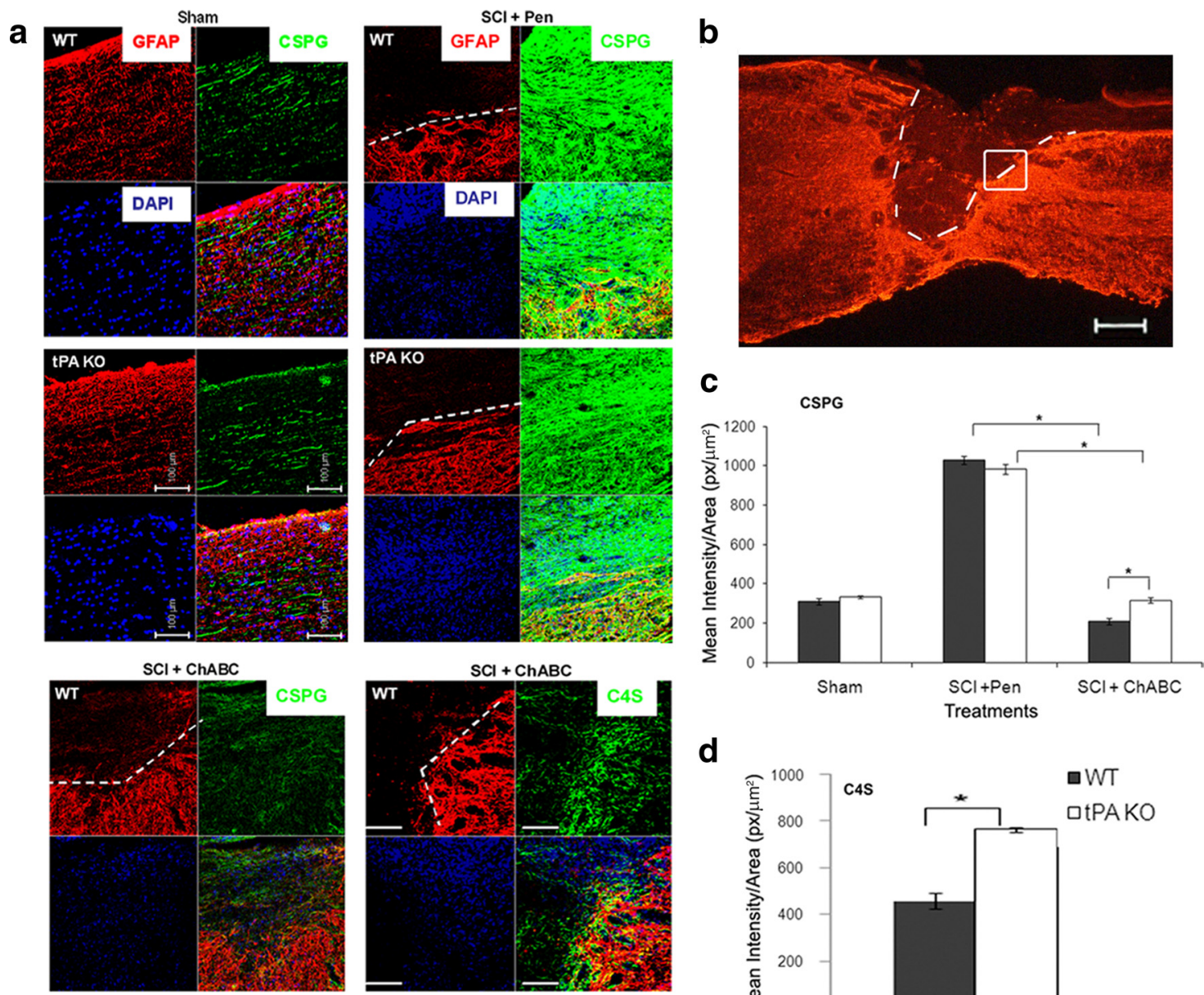

d
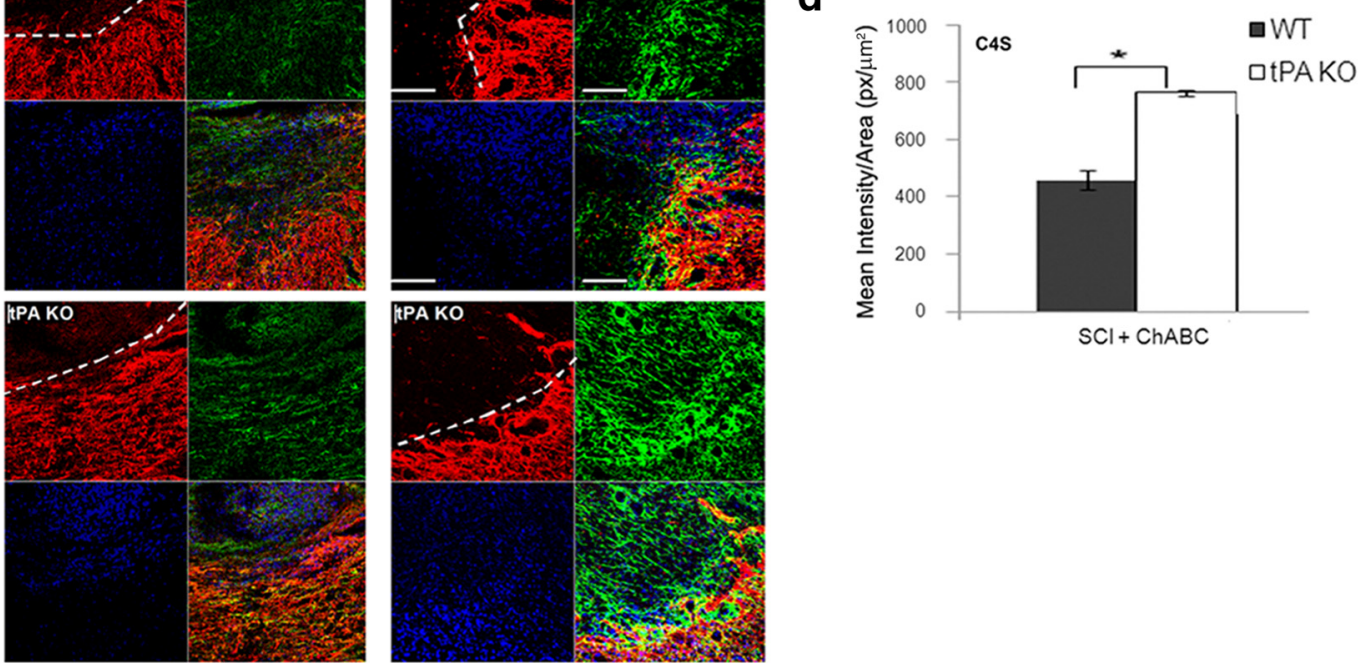

Figure 3. Higher levels of core CSPGs remain in PA KO animals even after ChABC cleavage in vivo. The $14 \mathrm{~d}$ sham or contusion-injured WT and tPA KO mice received Pen or ChABC injection ( 50 $\mathrm{U} / \mathrm{ml} \times 1 \mu \mathrm{l})$. Two days later spinal cords were isolated and perfused with PFA, and $18 \mu \mathrm{m}$ sagittal sections were prepared. $\boldsymbol{a}, \boldsymbol{b}, \mathrm{A} 14 \mathrm{~d}$ contusion-injured WT mouse spinal cord section stained with GFAP (red, DAKO) showing the injury region (dashed lines in $\boldsymbol{b}$ ) with the dorsal side up. Higher-magnification images were captured in $\boldsymbol{a}$ along the border of glial scar region as indicated by boxed area in $\boldsymbol{b}$. Scale bar, $200 \mu \mathrm{m}$. Spinal cord sections were then triple-stained for GFAP (red) and DAPI (blue, nuclear marker) and various CSPG protein antigens (in green, $\boldsymbol{a}$ ) CSPG (Sigma), C4S (Millipore Bioscience Research Reagents). Scale bar, $100 \mu \mathrm{m} . \boldsymbol{c}, \boldsymbol{d}$, ImageJ software (NIH) was used to calculate mean intensity/area for each group and protein staining around the injury border (identified by GFAP dead and reactive regions). $t$ test was used to compare within each treatment and one-way ANOVA to compare within genotype $(n=3-4)$. Significant ANOVA values were followed by post hoc Holm-Sidak. Brackets with asterisks indicate significance due to $t$ test or post hoc analyses with a minimum of $p<0.05$.

sive processes on spinal cord homogenates from intact WT and tPA KO mice, equivalent to cortical neurons grown in the absence of any substrate. Neurite outgrowth was significantly reduced on $14 \mathrm{~d}$ SCI homogenates from both genotypes treated with Pen in vitro. However, after in vitro ChABC treatment, significant neurite outgrowth was observed on the WT SCI homogenates $(p=0.031)$, but not on the TPA KO SCI homogenates ( $p<$ 0.001 ), albeit more on the latter than on the corresponding Pentreated SCI group ( $p=0.039$ ) (Fig. $5 d$ ). When plasmin was added in vitro to the tPA KO SCI homogenates with ChABC treatment, neurite outgrowth was restored $(p<0.001)$ and comparable to neurons grown on ChABC-treated WT SCI homogenates. In comparison, minimal levels of neurite outgrowth were evident on the Pen-plasmin-cotreated tPA KO SCI homogenate samples (Fig. $5 e, f$ ). Collectively, these results suggest that the $\mathrm{PA} / \mathrm{plasmin}$ pathway is an important contributor to neurite outgrowth after ChABC cleavage of CSPG GAG chains in an ex vivo glial scar environment.

\section{tPA/plasmin system plays a role in ChABC-mediated motor improvements}

The differences in CSPG accumulation after ChABC treatments in the tPA KO animals and the effect of the tPA/plasmin system on neurite outgrowth suggested that these cellular differences may translate to changes in behavior between WT and tPA KO mice after SCI. We used a set of behavioral assays in animals that 
a

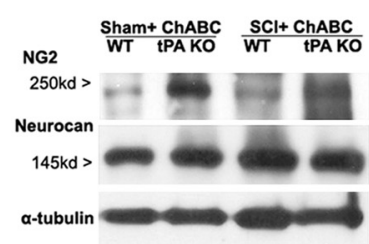

b

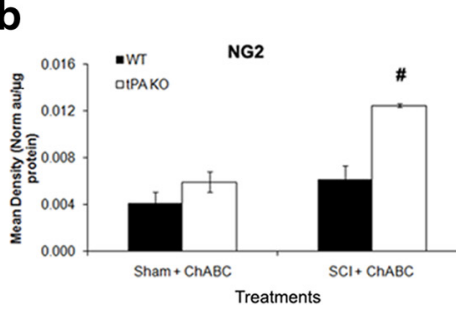

C
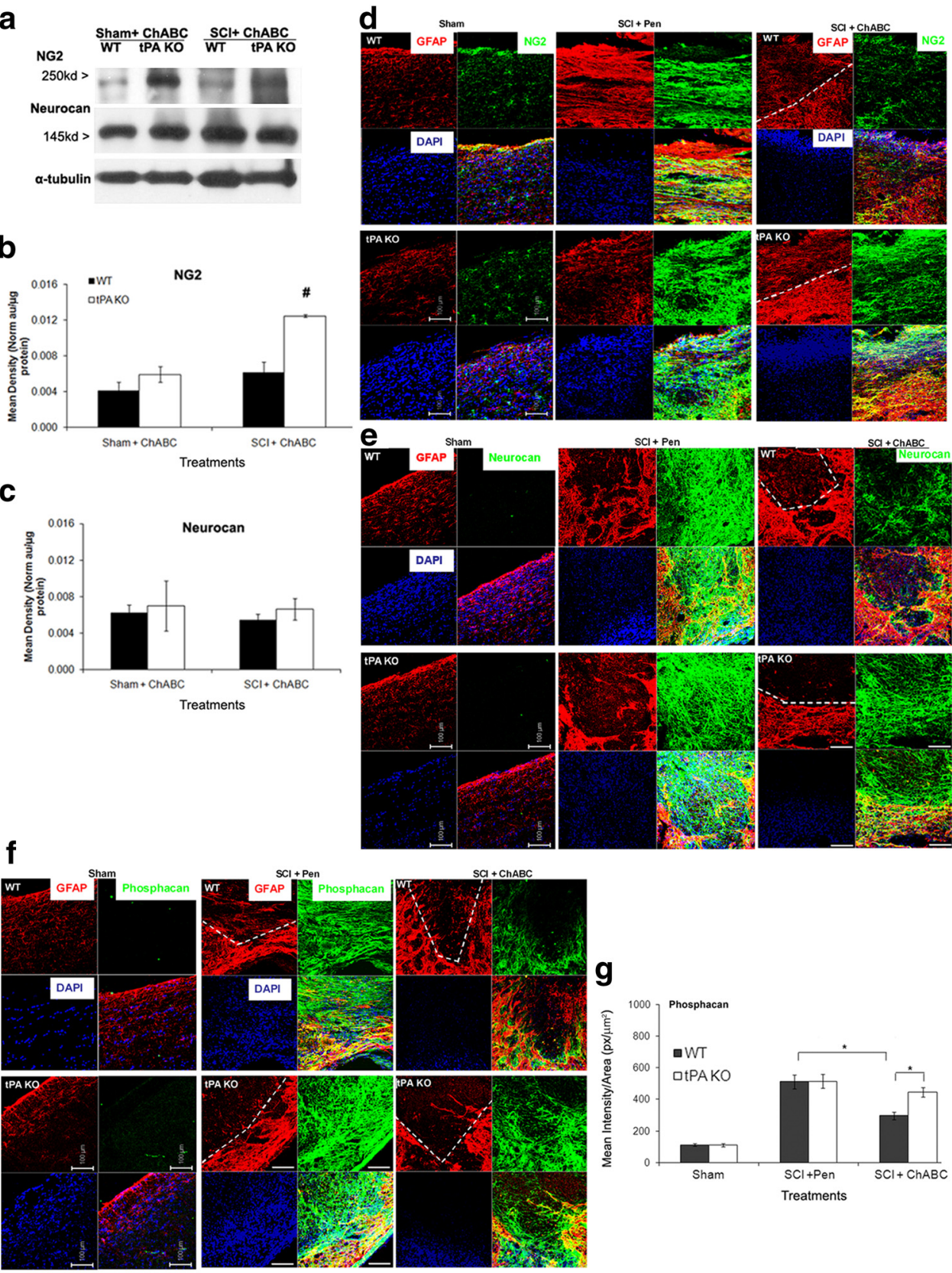

i

g
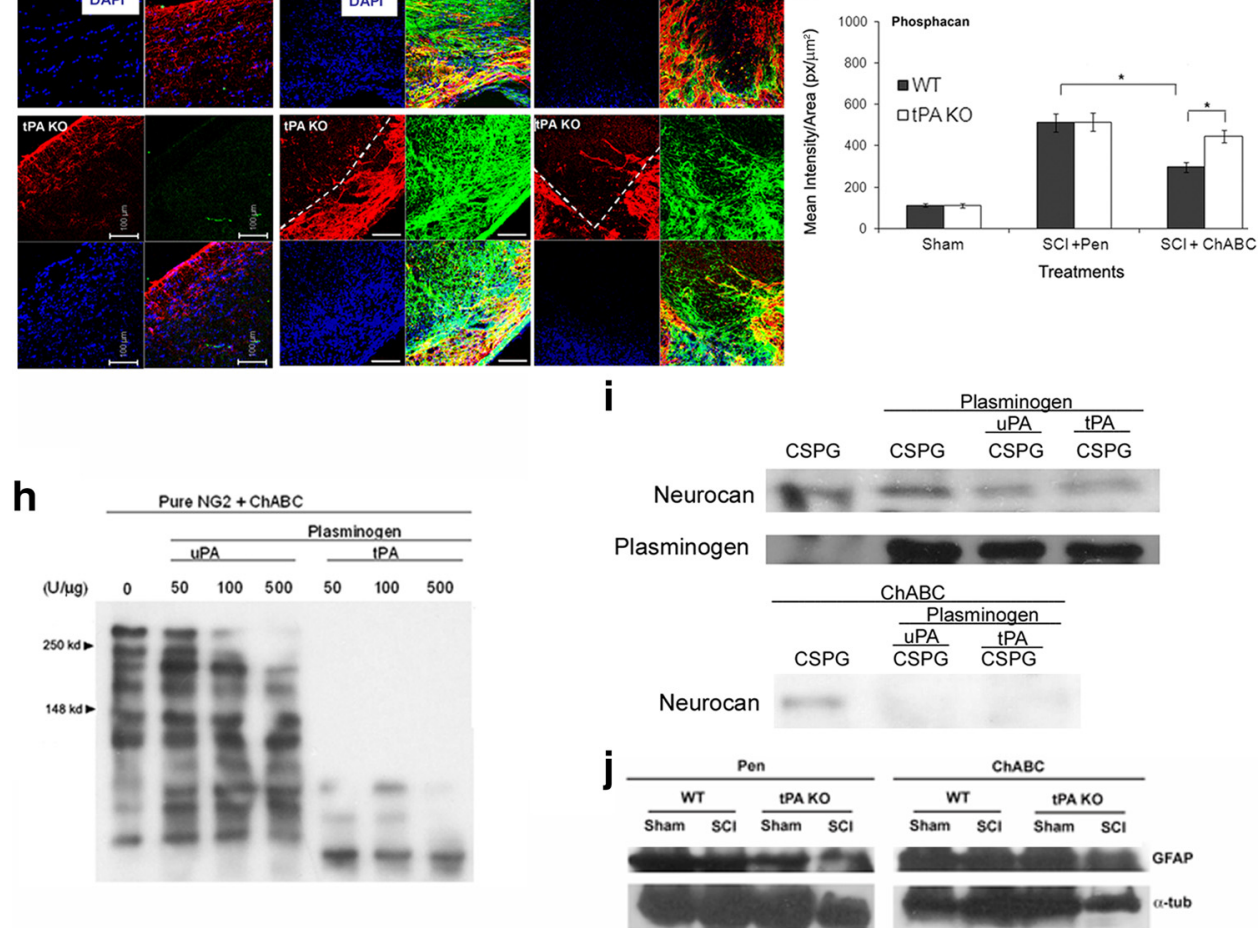

Figure 4. In the absence of tPA/plasmin system, NG2 and phosphacan core protein clearance is attenuated after ChABC cleavage in vivo. $\boldsymbol{a}$, Representative immunoblots of NG2 and neurocan protein expressions in WT and $\mathrm{TPA}$ KO sham and $14 \mathrm{dSCl}$ homogenates incubated with ChABC for $3 \mathrm{~h}$ at $37^{\circ} \mathrm{C}$ and probed with rabbit anti-NG2 and mouse anti-Neurocan antibodies. Protein expression could not be evaluated after Pen treatment in vitro using Western blotting since the presence of GAG chains resulted in a nondistinct protein band, data not shown. $\alpha$-Tubulin was used as a loading control.b, $\boldsymbol{c}$, Protein bands were quantified by densitometry in ImageJ and data normalized against $\alpha$-tubulin loading control and total protein levels. One-way ANOVA was (Figure legend continues.) 
had been subjected to SCI and were then treated with ChABC (or Pen) in vivo using the time course depicted in Figure 6. The accelerating rotor rod assay was used to measure motor balance in WT and tPA KO SCI mice. Differences due to genotype and time were first compared. Intact WT and tPA KO mice showed no differences in motor balance (Fig. 6a). In contrast, higher motor recovery was observed in $\mathrm{PA}$ KO SCI+Pen-treated mice at all time points after injury compared with the corresponding WT mice (Fig. 6b). This effect may possibly be due to the negative effect of tPA on the myelination process in WT SCI mice, as has been shown by other groups (Abe et al., 2003; Veeravalli et al., 2009). In the ChABC-treated SCI mice, no initial differences due to genotype were measured. However, WT SCI mice exhibited greater motor balance at post-ChABC injection time points compared with preinjection time points ( $p=0.003$ for day 18 vs day $7 ; p=0.004$ for day 18 vs day 10 ; and $p=0.004$ for day 21 vs day 7). tPA KO SCI- and ChABC-treated mice also showed improvements in motor balance at postinjection time point but not significantly different from preinjection time points (Fig. 6c). Differences due to treatment and time were then analyzed in two-way group comparisons holding genotype constant. WT SCI- and ChABC-treated mice showed significantly higher motor recovery at all postinjection time points compared with the same mice at preinjection time points and WT SCI- and Pen-treated group at postinjection time points $(p<0.05)$ (Fig. $6 d$ ). tPA KO SCI mice, however, did not show any differences due to ChABC treatment or time (Fig. 6e). Holding time constant, differences due to genotype and treatment were compared on day 21 after injury. Since genetic differences were found after SCI in the vehicle enzymetreated group compared with their genotypic equivalent WT controls, data were normalized to the Pen-treated groups. WT SCI and ChABC-treated mice showed higher motor balance compared with their genotypic equivalent Pen-treated group $(p<0.001)$ and tPA KO SCI and ChABC-treated mice $(p<0.05)$ (Fig. $6 f)$. These data suggest that $\mathrm{ChABC}$ treatment can lead to enhanced motor balance after SCI, and this motor balance improvement is not achieved in the absence of tPA/plasmin system.

Supported motor activity in an open field cage was also manually measured in all groups. WT and tPA KO intact mice exhib-

$\leftarrow$

(Figure legend continued.) used to compare all groups. Significant ANOVA was followed by post hoc Holm-Sidak test. Significant post hoc differences at ${ }^{\#} p<0.001(n=3)$. $\boldsymbol{d}-\boldsymbol{f}, 14 \mathrm{~d}$ sham or contusion-injured WT and TPA KO mice received Pen or ChABC injection $(50 \mathrm{U} / \mathrm{ml} \times 1$ $\mu$ l). Two days later, spinal cords were isolated and perfused with PFA, and $18 \mu \mathrm{m}$ sagittal sections were prepared. Spinal cord sections were then triple-stained for GFAP (red) and DAPI (blue, nuclear marker), and various (SPG protein antigens in green: NG2 (Millipore Bioscience Research Reagents) (b), phosphacan (3F8; DSHB) (d), and neurocan (1F6; DSHB) ( $\boldsymbol{f}$, and images were captured with a Zeiss confocal microscope using LSM 510 Meta Software. Dashed line indicates border of injury region. Images are representative of 3-4 biological replicates per group. Scale bar, $100 \mu \mathrm{m} . \boldsymbol{g}$, ImageJ software (NIH) was used to calculate mean intensity/area for each group around the injury border (identified by GFAP dead and reactive regions). A t test was used to compare within each treatment and one-way ANOVA to compare within genotype $(n=3-4)$. Significant ANOVA values were followed by posthoc Holm-Sidak test. Brackets with asterisks indicate significance due to $t$ test or post hoc analyses with a minimum of $p<0.05$.h, Western blot of NG2 protein (gift from Dr. Joel Levine) incubated with ChABC and treated with recombinant plasminogen and different concentrations of uPA or tPA for $3 \mathrm{~h}$ at $37^{\circ} \mathrm{C}$, followed by incubation with rabbit anti-NG2 antibody. ChABC cleaved NG2 protein can be seen at 250 and $148 \mathrm{kDa}$ (arrowheads). $\boldsymbol{i}$, Western blot of pure CSPG protein (CC117; Millipore Bioscience Research Reagents) incubated without (top blot) or with ChABC (bottom blot), pure plasminogen, $\mathrm{uPA}$, or tPA at $500 \mathrm{U} / \mu \mathrm{g}$ and probed for neurocan protein (1F6, DSHB). Plasminogen protein levels (anti-mouse plasminogen 1:1000, Millipore) were also assessed (middle blot).j, Western blot of sham or SCl protein extracts from WT and IPA KO animals treated with Pen or ChABC. The blots were probed with GFAP (Abcam), Iba1 (data not shown), and $\alpha$-tubulin. ited similar levels of high motor activity at all time points (Fig. $7 a$ ). All of the SCI groups demonstrated considerably lower levels of motor activity, confirming the effectiveness of the experimental SCI model (please note scale of $y$-axis, Fig. 7a-e). Two-way group comparisons between genotype and time were first analyzed. SCI and Pen-treated mice showed minimal motor recovery at preinjection and postinjection time points (Fig. $7 b$ ). SCI- and ChABC-treated groups exhibited improvement over time; at day 21 there was the highest recovery compared with days 7 and 10 ( $p=0.009$ and $p=0.010$ respectively) (Fig. $7 c$ ). Although no statistically significant differences due to treatment were measured, consistent trends were observed in-group comparisons for treatment and time (Fig. $7 d$ ). ChABC-treated injured mice showed levels of motor activity similar to the Pen-treated group at preinjection time points (day 10: $1.5 \pm 0.42$ vs $1.75 \pm 0.68$ counts), but much higher levels of motor activity at postinjection time points. On day 21, WT SCI + ChABC mice showed much better motor improvement than the Pen-treated group (10.0 \pm 3.65 vs $2.25 \pm 0.41$ counts). In some experiments a single high dose of plasmin was coadministered with Pen or ChABC to WT SCI mice only to determine whether ChABC-mediated motor recovery could be augmented and to evaluate the therapeutic potential of the tPA/plasmin mechanism. Data indicate that, if compared with the same animals at earlier time points and to the ChABC-treated group at the same time point, the ChABCplasmin-cotreated mice show a modest improvement in motor recovery on day 21 . Since day 21 appears to be the critical time point when all groups showed some level of motor recovery, behaviors were also compared across all genotypes and treatments on day 21 . When compared across treatments WT SCI mice showed evidence of motor recovery with the ChABC group displaying higher level of motor recovery compared with Penand Pen-plasmin-cotreated groups (444.4 vs 100.0 vs 222.2 ), but lower than ChABC-plasmin-cotreated groups (551.1 counts, Fig. $7 f)$. In contrast, the $\mathrm{PAA} \mathrm{KO} C \mathrm{ChBC}$ mice displayed lower motor recovery response (255.0). Overall, the supported motor activity data suggest that the tPA/plasmin pathway may play a role in ChABC-promoted motor recovery after SCI.

\section{tPA/plasmin system plays a role in ChABC-mediated sensory axonal plasticity}

Axon density was also quantified across all groups on day 22 after treatment at the glial scar region, and $800 \mu \mathrm{m}$ rostral and caudal to the injury. Data were then normalized to the axon density in the caudal region, as previously described (Tan et al., 2006, 2007). No significant differences were found between the sensory axons of intact WT and tPA KO mice across all three regions. WT SCI+Pen-treated mice showed decreased axon density in the glial scar $(27 \%)$ and rostral (26\%) regions compared with the caudal region respectively. Treatments with Pen + plasmin, ChABC $(p=0.002)$ and ChABC + plasmin $(p<0.001)$ resulted in increases in axonal density in the glial scar region compared with the SCI mice treated with Pen alone (Fig. 8). However, in the rostral region, only the $\mathrm{ChABC}+$ plasmin-treated WT SCI mice $(p<0.001)$ demonstrated significantly higher axon density compared with the Pen-treated WT SCI mice. These data suggest that deglycosylation of CSPGs by ChABC primarily affects axonal regrowth in the local glial scar environment with only minor effects in the nearby rostral region. Plasmin, generated by tPA's action on plasminogen, can similarly cleave CSPGs within the glial scar region regardless of their glycosylation status, but appears to be only minimally effective in the rostral region. However, the cotreatments of ChABC and plasmin can augment the axonal den- 

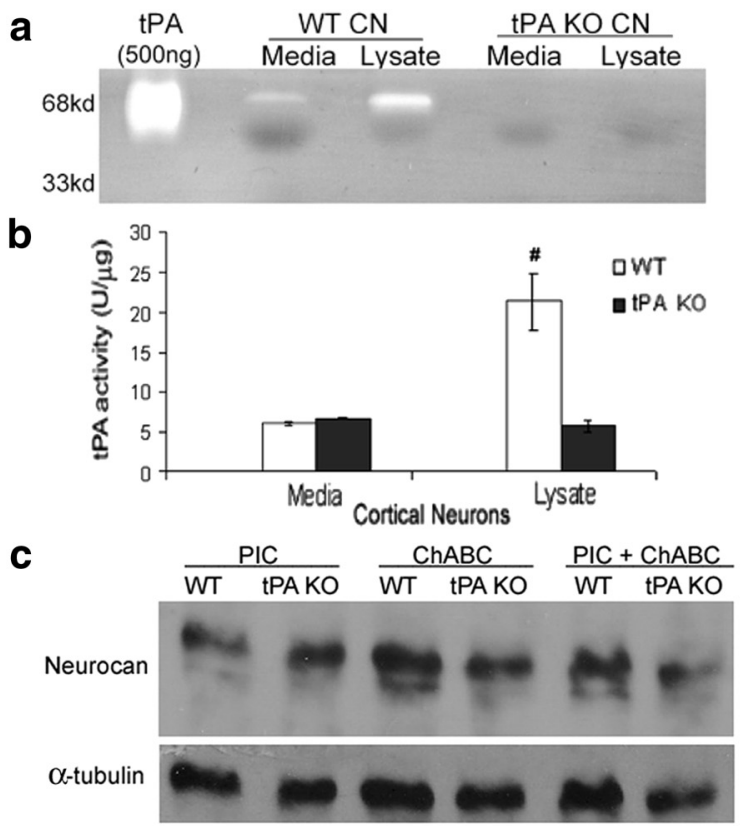

e
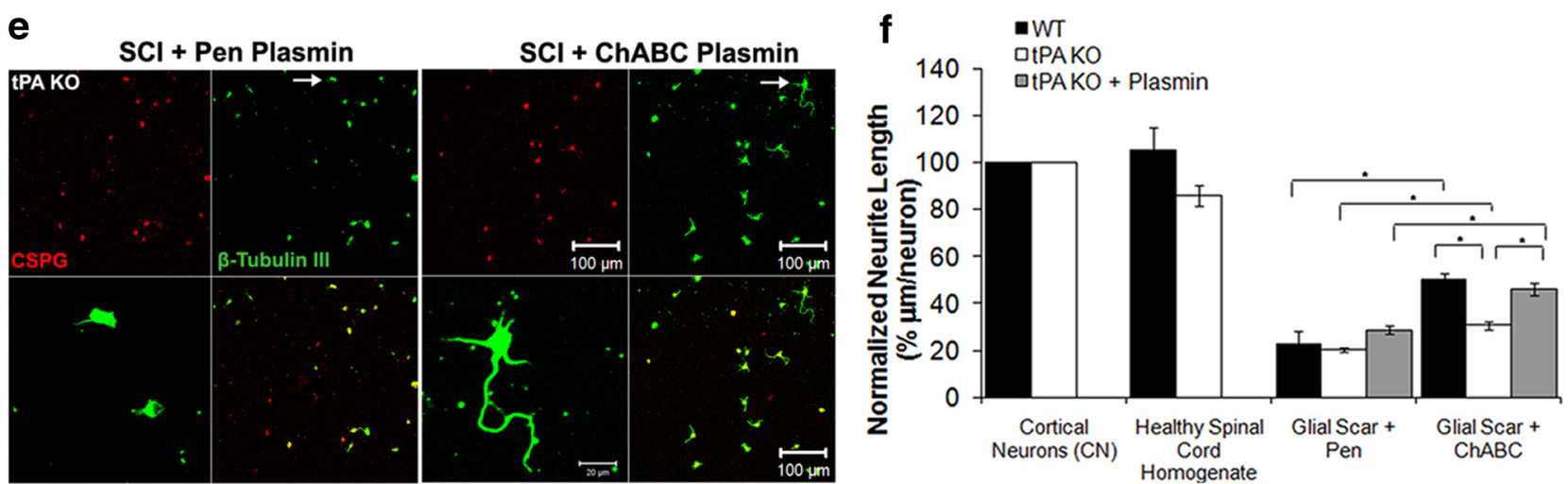

Figure 5. TPA/plasmin system allows for enhanced neurite outgrowth after ChABC cleavage of CSPGs in ex vivo glial scar. $\boldsymbol{a}, \boldsymbol{b}$, tPA activity was visualized in media and cell lysates from embryonic day 15 WT and tPA KO cortical neurons using zymography $(\boldsymbol{a})$, and measured using quantitative amidolytic assay ( $\boldsymbol{b})$. Pure recombinant tPA was used as positive control and can be seen at $68 \mathrm{kDa}$ in $\boldsymbol{a}$. Significantly higher levels of tPA activity were measured in WT cortical neuron cell lysates compared with all other groups ( $n=3$ ). One-way ANOVA was used to compare all groups. Significant ANOVA was followed by post hoc Holm-Sidak test. Significant post hoc differences at ${ }^{\#} p<0.001$. c, Western blot of $14 \mathrm{~d}$ SCl extracts from WT and tPA K0 mice incubated with protease inhibitor cocktail (PIC) (1X; Sigma), and/or ChABC ( $0.5 \mathrm{U} / \mathrm{ml})$ for $3 \mathrm{~h}$ at $37^{\circ} \mathrm{C}$ and probed for Neurocan protein and $\alpha$-tubulin. $\boldsymbol{d}$, Spinal cord homogenates from sham or $14 \mathrm{~d}$ contusion-injured mice were treated with Pen or $\mathrm{ChABC}(0.5 \mathrm{U} / \mathrm{ml})$ in vitro for $3 \mathrm{~h}$ at $37^{\circ} \mathrm{C}$. Embryonic day $15 \mathrm{WT}$ and tPA KO cortical neurons were then plated on spinal cord homogenates of the same genotype. Two days later, cultures were fixed with PFA and stained for intact CSPG (red; CS56; Sigma), and $\beta$-tubulin III (green; Tuj1; Covance) Scale bar, $100 \mu \mathrm{m}$. Higher-magnification images of a neuron in each group (indicated by an arrow in $\beta$-tubulin III staining) are provided in the lower left box of each image panel. Scale bar, $20 \mu \mathrm{m} . \boldsymbol{e}$, Embryonic day 15 tPA K0 cortical neurons were grown on $14 \mathrm{~d}$ contusion-injured tPA KO SCl homogenates previously cotreated with Pen and plasmin or ChABC and plasmin for $3 \mathrm{~h}$ at $37^{\circ} \mathrm{C}$. Two days later, cultures were fixed with PFA and stained for intact CSPG (red), and $\beta$-tubulin III (green). Scale bar, $100 \mu \mathrm{m}$. Higher-magnification image of a neuron in each group (indicated by an arrow in $\beta$-tubulin III staining) is provided in the lower left box of each image panel. tPA KO cortical neurons grown on IPA KO SCI homogenates cotreated with ChABC and plasmin in vitro showed significantly higher neurite outgrowth compared with neurons grown on tPA KO SCI homogenates cotreated with Pen and plasmin. Scale bar, $20 \mu \mathrm{m}$. Images are representative of 3 biological replicates per group. $f$, NeuriteTracer plug-in in ImageJ was used to quantify $400-600$ neurons per biological replicate. Data were calculated as neurite length/neuron, normalized and plotted as a percentage of the cortical neuron only group of the same genotype $(n=3)$. One-way ANOVA was used for comparisons. Significant ANOVA was followed by post hoc Holm-Sidak test. Brackets with asterisk indicate significant post hoc differences. Compared with neurite outgrowth on SCl homogenates from WT and tPA KO mice treated with Pen in vitro, $p=0.031$ and $p=0.039$ for neurite outgrowth on the same SCI homogenate samples from WT and tPA KO mice treated with ChABC in vitro. When compared within ChABC-treated group, cortical neurons on WT SCI homogenates grew more processes than cortical neurons on $\mathrm{PAA}$ KO SCI homogenates ( $p<$ 0.001). Furthermore, tPA KO cortical neurons grown on tPA KO SCI homogenates treated with ChABC and plasmin ( $0.06 \mathrm{U} / \mathrm{ml})$ in vitro show significantly higher neurite outgrowth when compared with neurons on TPA KO SCI homogenates treated with ChABC $(p<0.001)$ or Pen Plasmin in vitro $(p=0.002)$.

sity in both the glial scar and rostral regions of the spinal cord suggesting that the two enzymes may be acting synergistically to enhance axonal outgrowth both within and beyond the glial scar region.

In contrast, the axonal density within the glial scar region of tPA KO SCI+Pen-treated mice was at $60 \%$ axon density of that in the caudal region, potentially due to a lesser degree of axonal retraction and dieback (Kerschensteiner et al., 2005). Treatment with Pen + plasmin or ChABC, however, did not significantly im- prove the axonal density. Cotreatment with $\mathrm{ChABC}+$ plasmin resulted in partial recovery of axonal density to $20 \%$ over the Pen-treated group. In the rostral region, the axonal density in $\mathrm{PAA}$ KO SCI+Pen-treated mice was only $20 \%$ of that of the caudal region and the addition of $\mathrm{ChABC}$ does not improve this response. The addition of plasmin, however, significantly enhanced axonal density to $59 \%$ and was not different from the axonal density changes observed with $\mathrm{ChABC}+$ plasmin-cotreated mice $(p<0.001)$. 

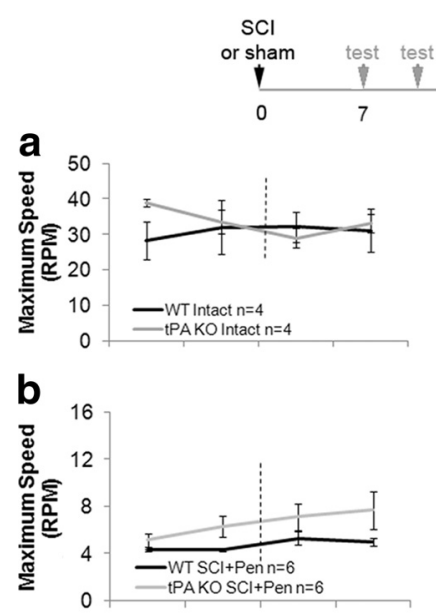

ChABC
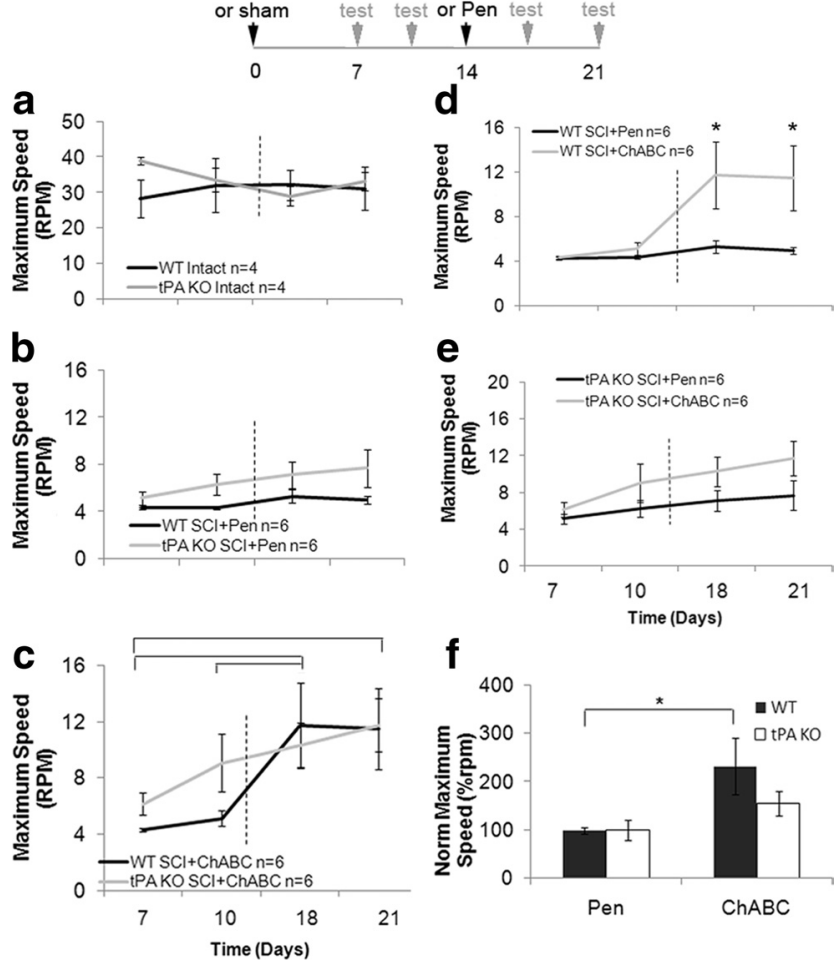

Figure 6. ChABC promoted motor balance is attenuated in the absence of $\mathrm{tPA} /$ plasmin system. WT and PA KO mice underwent sham or contusion injury followed by a single intraspinal injection of $\mathrm{ChABC}(50 \mathrm{U} / \mathrm{ml})$ or Pen on day 14 . An accelerating rotor rod was used to measure mice's motor balance on designated days and the maximum speed the mice remained on the machine was recorded. Two-way group comparisons are presented in graphs $\boldsymbol{a}-\boldsymbol{e}$ with graphs $\boldsymbol{a}-\boldsymbol{c}$ comparing differences due to genotype and time, while graphs $\boldsymbol{d}$ and $\boldsymbol{e}$ illustrate differences due to treatment and time. Two-way repeated-measures ANOVA was used for multiple group comparisons. Significant ANOVA was followed by post hoc Holm-Sidak test. Brackets in $\mathrm{c}$ indicate significantly higher recovery in WT SCI+ChABC mice on day 18 compared with day 7 $(p=0.003)$ and day $10(p=0.004)$, and significantly higher recovery on day 21 compared with day $7(p=0.004)$. Asterisks in $\boldsymbol{d}$ indicate significantly higher recovery in WT SCI+ChABC mice compared with WT SCl+Pen group on day $18(p<0.001)$ and day $21(p=0.001)$. $\boldsymbol{f}$, Motor coordination differences due to genotype and treatment were compared on day $21(n=3)$. At test was used to compare between treatments and genotypes. Brackets with asterisks indicated significant difference between WT SCI+ChABC and WT SCI + Pen groups $(p<0.001)$ and WT $\mathrm{SCl}+\mathrm{ChABC}$ and $\mathrm{PAAKOSCl}+\mathrm{ChABC}$ groups $(p=0.02)$.

\section{Discussion}

A survival analysis of all our SCI mice revealed a moderate injury severity with no significant variability between genotypes or treatments. As a result, any differences between groups were due to the specific effect of genotype or treatment on injury rather than any technical differences between groups. Although the mechanism or robustness of the deglycosylation activity of ChABC is not established, we were able to quantify a significant effect of the enzyme on CSPG proteins, which resulted in promoting neurite outgrowth on glial scar homogenates, improving motor activity and enhancing sprouting of sensory axons in the glial scar regions after contusion injury and enzyme treatment. We, therefore, were able to reproduce improvements in recovery promoted by $\mathrm{ChABC}$ in the glial scar region as demonstrated by other groups (Bradbury et al., 2002; Caggiano et al., 2005; Barritt et al., 2006; Cafferty et al., 2008).

Our early studies led to the hypothesis that GAG chain removal by ChABC not only removes an inhibitory component of CSPG molecules but also facilitates tPA/plasminogen binding to the inhibitory core protein of CSPG molecules (Nolin et al.,

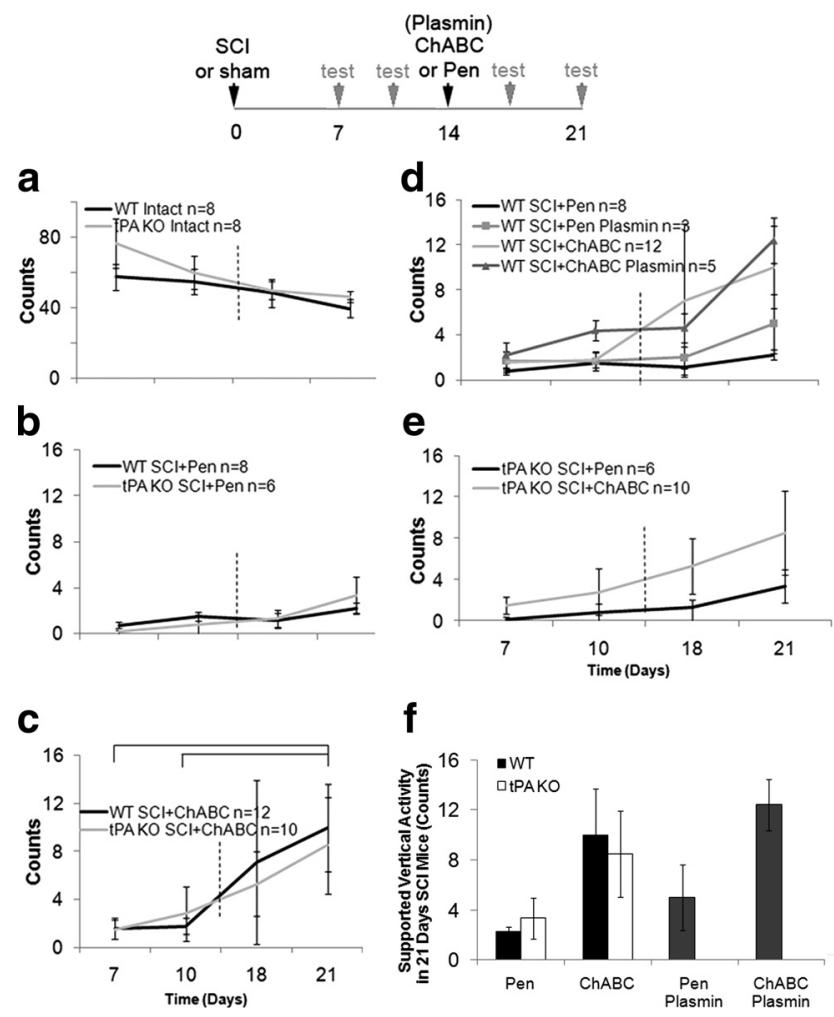

Figure 7. ChABC promoted supported motor recovery is only mildly affected in the absence of tPA/plasmin system. WT and tPA KO mice underwent sham and contusion injury followed by a single Pen, or ChABC injection on day 14. WT SCI mice also received cotreatments of Pen + plasmin or $C h A B C+$ plasmin. Mice were placed in an open field cage and supported motor activity in a 20 min observation period was manually recorded on designated days. Two-way group comparisons are presented with graphs $\boldsymbol{a}-\boldsymbol{c}$ comparing differences due to genotype and time, while graphs $\boldsymbol{d}$ and $\boldsymbol{e}$ illustrate differences due to treatment and time. $\boldsymbol{f}$, Supported motor activity differences due to genotype and treatment were compared on day 21. Unfortunately, due to the large intragroup variability, the study remains underpowered.

2008). These core proteins then act as a scaffold for the localized formation of more plasmin which, in turn, can target and degrade the inhibitory core proteins and allow for anatomical and behavioral improvements. Our results now propose that the tPA/plasmin pathway may specifically bind after ChABC cleavage of GAG chains and degrade structurally unique CSPG core proteins, NG2 and phosphacan, and nonspecifically bind to the larger and structurally homologous family of lecticans of which neurocan is a member. Clearance of these inhibitory core CSPG proteins by the tPA/plasmin system may partially contribute to enhanced neurite outgrowth.

The function of NG2, specifically, in neurite outgrowth has been discussed by various groups, either indicating an inhibitory core and holoprotein (Dou and Levine, 1994; Ughrin et al., 2003), or suggesting that NG2 does not inhibit neurite outgrowth (Hossain-Ibrahim et al., 2007; Busch et al., 2010). It is conceivable that the difference lies in the form of NG2 evaluated (cellassociated vs deposited). However, our data suggest that it is the extracellular deposition of multiple CSPGs that contribute to the inhibitory environment.

Our results also demonstrate that $\mathrm{ChABC}$ facilitated improvements in neurite sprouting in the glial scar region and motor recovery can be attenuated in the absence of the tPA/plasmin system. Similarly, treatment with plasmin, the end-protease of the $\mathrm{tPA} /$ plasmin cascade can allow for some improvements in motor recovery and neurite outgrowth in the glial scar region. 


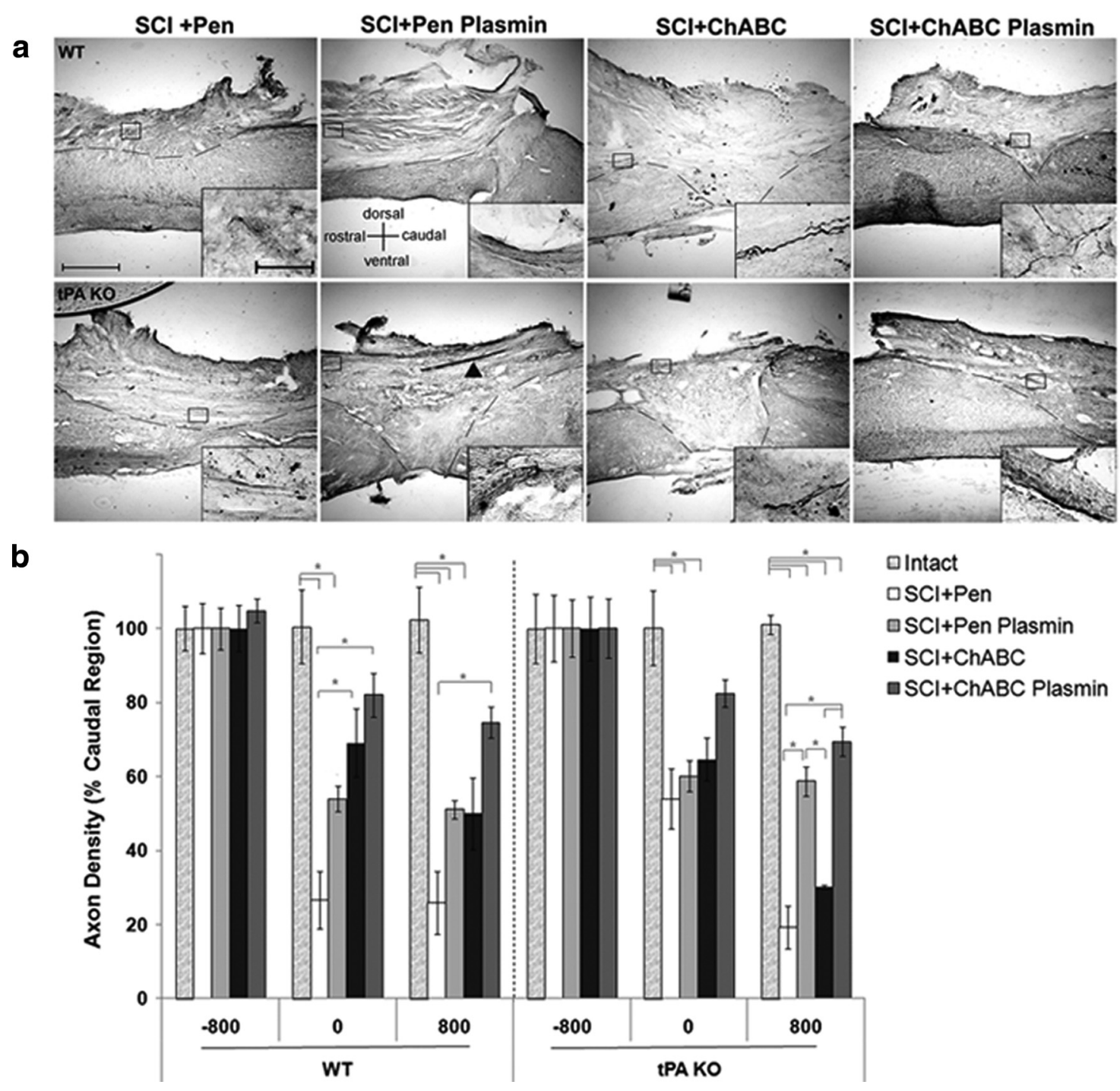

Figure 8. ChABC and plasmin cotreatments significantly enhance synaptic plasticity of sensory axons in the glial scar regions. WT and tPA KO mice underwent sham or contusion injury and a single injection of Pen, ChABC, Pen + plasmin or ChABC + plasmin injection on day 14. On day 22, 1.5\% CTB was injected into the mice's sciatic nerve and spinal cord isolated $4 \mathrm{~d}$ later. Sagittal spinal cord sections $(20 \mu \mathrm{m})$ were then stained with CTB-HRP followed by DAB and silver-gold intensification reaction. Images were captured on Nikon E600 microscope with Nis-Elements software and oriented with dorsal side up and rostral end on the left. $\boldsymbol{a}$, Injured region in each spinal cord section is indicated by dashed lines at low magnification and a representative axon in each injury region boxed and shown in a higher-magnification image on the bottom right of each image panel. Arrowhead in TPA KO SCI+Pen Plasmin image panel points to remnants of a suture. Scale bar, $500 \mu \mathrm{m}$ in low-magnification panel and $50 \mu \mathrm{m}$ in high-magnification panel. $\boldsymbol{b}$, Stereoinvestigator software was used to quantify axon density in the glial scar region $(0 \mu \mathrm{m})$, rostral $(-800 \mu \mathrm{m})$ and caudal regions $(800 \mu \mathrm{m})$ in each spinal cord and data normalized and plotted as a percentage of axon density in the caudal region of each spinal cord $(n=3-4)$. One-way ANOVA was used for comparisons within each region and genotype. Significant ANOVA was followed by post hoc Holm-Sidak test. Brackets with asterisk indicate significant post hoc differences. Compared with WT intact mice, WT $\mathrm{SCl}+$ Pen and WT SCI + Pen plasmin mice show significantly less axon density $(p<0.001$ and $p=0.002$ respectively) in the glial scar region. WT SCl and $\mathrm{ChABC}$ and ChABC + plasmin mice show significantly higher axon density $(p=0.002$ and $p<0.001)$ compared with WTSCI + Pen mice in the glial scar region. In the rostral region, WTSCI + Pen-, Pen + plasmin-, and ChABC-treated mice showed significantly lower axon density $(p<0.001)$ compared with WT intact mice. ChABC + plasmin-cotreated mice showed significantly higher axon density compared with pen alone-treated mice $(p<0.001)$. In the PA KO SCI mice, Pen alone, Pen + plasmin, and ChABC treatments resulted in significantly lower axon density $(p=0.012, p=0.001, p=0.002$ respectively) compared with tPA KO intact mice in the glial scar region. In contrast, in the rostral region, all treatment groups had significantly lower axon density compared with intact tPA K0 mice ( $p<0.001)$. Both Pen + plasmin and ChABC + plasmin-treated mice showed significantly higher axon density compared with Pen alone $(p<0.001)$ and ChABC alone treatments $(p<0.001)$.

However, the cotreatment of ChABC and plasmin in both WT and tPA KO SCI mice in the glial scar region resulted in improvements that exceeded each individual treatment alone and extended outgrowth into the neighboring rostral region of the spinal cord. Interestingly, treatment with plasmin in the tPA KO SCI mice resulted in minimal neurite outgrowth and ChABC+ plasmin cotreatments in the tPA KO SCI mice also allowed for only a partial rescue response. This result suggests that although plasmin is the end product of the tPA/plasmin cascade, it may still require tPA to affect synaptic plasticity in the glial scar region. Indeed in a model where tPA KO or plasmin(ogen) KO neurons were cultured on CNS myelin membranes, the absence of plasmin (ogen) minimally affected neurite outgrowth, whereas the deficiency of tPA was critical for outgrowth (Minor et al., 2009), suggesting the presence of additional nonproteolytic function for tPA. Therefore, although ChABC and tPA/plasmin system can allow for improvements in functional motor recovery independently, we propose that the collective clearance of inhibitory GAG chains and inhibitory core proteins by $\mathrm{ChABC}$ and tPA/plasmin system create a synergistic relationship that contributes to a more permissive environment resulting in enhanced synaptic plasticity and motor recovery than each treatment alone. 
Our results also consistently showed that tPA KO SCI- and vehicle enzyme-treated mice showed greater synaptic plasticity through motor and sensory recovery in the glial scar region than the corresponding WT SCI mice. Two groups have also reported similar results attributing the recovery to an interaction between tPA/microglia and myelin basic protein which produces greater demyelination (Abe et al., 2003; Veeravalli et al., 2009) and does not involve tPA's proteolytic activity.

Our data also suggest a role for the $\mathrm{uPA} /$ plasmin system in SCI relevant to ChABC-promoted synaptic plasticity. uPA is not normally found in the (Tsirka et al., 1997) but after SCI, the blood brain barrier breakdown allows more uPA to relocate into the spinal cord. As a result, in WT intact mice spinal cord homogenates uPA activity was lower than tPA activity. In contrast, after injury, similar levels of tPA and UPA activity were measured in WT contusion-injured mice, and uPA activity was found to be significantly upregulated after $14 \mathrm{~d}$ injury in tPA KO SCI homogenates. The $\mathrm{uPA} / \mathrm{plasmin}$ system also interacted with pure neurocan after ChABC cleavage of GAG chains in vitro and allowed for clearance of the core protein suggesting a potential role for uPA in binding and degrading the structurally homologous lectican family of CSPG. Based on these findings, it would be worth investigating whether part of the residual motor recovery and synaptic plasticity observed in ChABC-treated tPA KO SCI mice can be attributed to uPA/plasmin compensatory degradation. Therefore, similar to the tissue remodeling seen in the crossed phrenic phenomenon of respiratory function following SCI (Minor and Seeds, 2008; Seeds et al., 2009), it would be interesting to determine whether both plasminogen activators play a role in clearance of CSPG core proteins after SCI and additionally assess plasminogen-independent roles for the plasminogen activators (Minor et al., 2009).

Following contusion injury, the biochemical characterization revealed an upregulation of overall serine protease activity. Since we and others (Veeravalli et al., 2009) have shown that plasminogen activators are chronically elevated, the presence and upregulation of other serine protease activities at acute and subacute time points after SCI require further studies. One such early study reported an increase in neurotoxic prothrombin and its receptor protease-activated receptor gene family (PAR-1) beginning as early as $8 \mathrm{~h}$ and reaching a maximal level at $24 \mathrm{~h}$ in a rat contusion injury model (Citron et al., 2000). Furthermore, using gene array analyses of SCI tissue, they found that several serine proteases were activated by SCI underscoring the need for further characterization of serine protease activities at acute time points after injury.

The data obtained from the sensory axon density measurements suggest that $\mathrm{PA}$ may function within the SCI environment both in a proteolysis-independent role affecting demyelination, and in a proteolysis-dependent role resulting in the clearance of CSPG core protein. Cotreatment with ChABC and plasmin can partially rescue the $\mathrm{tPA}$-dependent axonal density further demonstrating that the $\mathrm{tPA} /$ plasmin cascade is functioning downstream of $\mathrm{ChABC}$ in the local glial scar environment. Interestingly although plasmin is the end-protease in the $\mathrm{tPA}$ /plasmin system, it seems that it still requires the presence of tPA in the glial scar region to allow for improvements in synaptic plasticity, as the absence of tPA mitigates both the plasmin alone-mediated axonal density and $\mathrm{ChABC}+$ plasmincotreated axonal density in PAA KO SCI mice.

Although the absence of tPA did not result in dramatic changes in the behavioral results (potentially due to compensation by other proteases), the introduction of plasmin appeared to enhance the outcomes both in behavioral tests and in the anatomical assessment. In our study we have used one paradigm of plasmin delivery and it would be critical to evaluate different modes of delivery, timing and doses.

It is not clear whether the functions of tPA are dependent on the distance from the glial scar. It is possible then that while ChABC contributes to synaptic plasticity in the glial scar region, tPA affects synaptic plasticity both through proteolysis-dependent and -independent roles in the lesion area. This would be consistent with a nonproteolytic role of tPA associated with activated microglia (Siao and Tsirka, 2002) which would localize mostly in the lesion epicenter. Collectively, our results suggest that ChABC degradation of the GAG chains not only removes an inhibitory component of CSPG molecules but may also facilitate tPA/plasmin(ogen)'s interaction with the inhibitory core proteins in vivo. The close proximity of tPA and plasminogen proteins allows for formation of more plasmin which in turn may enhance clearance of the inhibitory core CSPG proteins. Although ChABC cleavage of GAG chains and plasmin degradation of core proteins can independently contribute to synaptic plasticity, our results also demonstrate that the synergistic interaction of both on CSPG proteins in vivo may allow for greater improvements in synaptic plasticity and motor recovery than either treatment alone.

\section{References}

Abe Y, Nakamura H, Yoshino O, Oya T, Kimura T (2003) Decreased neural damage after spinal cord injury in tPA-deficient mice. J Neurotrauma 20:43-57.

Barritt AW, Davies M, Marchand F, Hartley R, Grist J, Yip P, McMahon SB, Bradbury EJ (2006) Chondroitinase ABC promotes sprouting of intact and injured spinal systems after spinal cord injury. J Neurosci 26:10856-10867.

Bradbury EJ, Moon LD, Popat RJ, King VR, Bennett GS, Patel PN, Fawcett JW, McMahon SB (2002) Chondroitinase ABC promotes functional recovery after spinal cord injury. Nature 416:636-640.

Busch SA, Horn KP, Cuascut FX, Hawthorne AL, Bai L, Miller RH, Silver J (2010) Adult NG2 + cells are permissive to neurite outgrowth and stabilize sensory axons during macrophage-induced axonal dieback after spinal cord injury. J Neurosci 30:255-265.

Cafferty WB, Bradbury EJ, Lidierth M, Jones M, Duffy PJ, Pezet S, McMahon SB (2008) Chondroitinase ABC-mediated plasticity of spinal sensory function. J Neurosci 28:11998-12009.

Caggiano AO, Zimber MP, Ganguly A, Blight AR, Gruskin EA (2005) Chondroitinase $\mathrm{ABCI}$ improves locomotion and bladder function following contusion injury of the rat spinal cord. J Neurotrauma 22:226-239.

Citron BA, Smirnova IV, Arnold PM, Festoff BW (2000) Upregulation of neurotoxic serine proteases, prothrombin, and protease-activated receptor 1 early after spinal cord injury. J Neurotrauma 17:1191-1203.

Collen D (1999) The plasminogen (fibrinolytic) system. Thromb Haemost $82: 259-270$.

Davies JE, Tang X, Denning JW, Archibald SJ, Davies SJ (2004) Decorin suppresses neurocan, brevican, phosphacan and NG2 expression and promotes axon growth across adult rat spinal cord injuries. Eur J Neurosci 19:1226-1242.

Davies JE, Tang X, Bournat JC, Davies SJ (2006) Decorin promotes plasminogen/plasmin expression within acute spinal cord injuries and by adult microglia in vitro. J Neurotrauma 23:397-408.

Davies SJ, Fitch MT, Memberg SP, Hall AK, Raisman G, Silver J (1997) Regeneration of adult axons in white matter tracts of the central nervous system. Nature 390:680-683.

Dou CL, Levine JM (1994) Inhibition of neurite growth by the NG2 chondroitin sulfate proteoglycan. J Neurosci 14:7616-7628.

Fulmer T (2009) Unblocking axonal regeneration. Sci Bus Exchange 2:1588-1590.

Hellal F, Hurtado A, Ruschel J, Flynn K, Laskowski CJ, Umlauf M, Kapitein LC, Strikis D, Lemmon V, Bixby J, Hoogenraad CC, Bradke F (2011) Microtubule stabilization reduces scarring and causes axon regeneration after spinal cord injury. Science 331:928-931. 
Hossain-Ibrahim MK, Rezajooi K, Stallcup WB, Lieberman AR, Anderson PN (2007) Analysis of axonal regeneration in the central and peripheral nervous systems of the NG2-deficient mouse. BMC Neurosci 8:80.

Ito Y, Hikino M, Yajima Y, Mikami T, Sirko S, von Holst A, Faissner A, Fukui S, Sugahara K (2005) Structural characterization of the epitopes of the monoclonal antibodies 473HD, CS-56, and MO-225 specific for chondroitin sulfate D-type using the oligosaccharide library. Glycobiology 15:593-603.

Jones LL, Yamaguchi Y, Stallcup WB, Tuszynski MH (2002) NG2 is a major chondroitin sulfate proteoglycan produced after spinal cord injury and is expressed by macrophages and oligodendrocyte progenitors. J Neurosci 22:2792-2803.

Jones LL, Margolis RU, Tuszynski MH (2003) The chondroitin sulfate proteoglycans neurocan, brevican, phosphacan, and versican are differentially regulated following spinal cord injury. Exp Neurol 182:399-411.

Kerschensteiner M, Schwab ME, Lichtman JW, Misgeld T (2005) In vivo imaging of axonal degeneration and regeneration in the injured spinal cord. Nat Med 11:572-577.

Laabs TL, Wang H, Katagiri Y, McCann T, Fawcett JW, Geller HM (2007) Inhibiting glycosaminoglycan chain polymerization decreases the inhibitory activity of astrocyte-derived chondroitin sulfate proteoglycans. J Neurosci 27:14494-14501.

McKeon RJ, Schreiber RC, Rudge JS, Silver J (1991) Reduction of neurite outgrowth in a model of glial scarring following CNS injury is correlated with the expression of inhibitory molecules on reactive astrocytes. J Neurosci 11:3398-3411.

Minor KH, Seeds NW (2008) Plasminogen activator induction facilitates recovery of respiratory function following spinal cord injury. Mol Cell Neurosci 37:143-152.

Minor K, Phillips J, Seeds NW (2009) Tissue plasminogen activator promotes axonal outgrowth on CNS myelin after conditioned injury. J Neurochem 109:706-715.

Moon LD, Asher RA, Rhodes KE, Fawcett JW (2001) Regeneration of CNS axons back to their target following treatment of adult rat brain with chondroitinase ABC. Nat Neurosci 4:465-466.

Morgenstern DA, Asher RA, Fawcett JW (2002) Chondroitin sulphate proteoglycans in the CNS injury response. Prog Brain Res 137:313-332.

Niederöst BP, Zimmermann DR, Schwab ME, Bandtlow CE (1999) Bovine CNS myelin contains neurite growth-inhibitory activity associated with chondroitin sulfate proteoglycans. J Neurosci 19:8979-8989.

Nishiyama A, Dahlin KJ, Prince JT, Johnstone SR, Stallcup WB (1991) The primary structure of NG2, a novel membrane-spanning proteoglycan. J Cell Biol 114:359-371.

Nolin WB, Emmetsberger J, Bukhari N, Zhang Y, Levine JM, Tsirka SE (2008) tPA-mediated generation of plasmin is catalyzed by the proteoglycan NG2. Glia 56:177-189.

Oohira A, Matsui F, Katoh-Semba R (1991) Inhibitory effects of brain chon- droitin sulfate proteoglycans on neurite outgrowth from PC12D cells. J Neurosci 11:822-827.

Pool M, Thiemann J, Bar-Or A, Fournier AE (2008) NeuriteTracer: a novel ImageJ plugin for automated quantification of neurite outgrowth. J Neurosci Methods 168:134-139.

Seeds NW, Akison L, Minor K (2009) Role of plasminogen activator in spinal cord remodeling after spinal cord injury. Respir Physiol Neurobiol 169:141-149.

Siao CJ, Tsirka SE (2002) Tissue plasminogen activator mediates microglial activation via its finger domain through annexin II. J Neurosci 22:3352-3358.

Smith-Thomas LC, Fok-Seang J, Stevens J, Du JS, Muir E, Faissner A, Geller HM, Rogers JH, Fawcett JW (1994) An inhibitor of neurite outgrowth produced by astrocytes. J Cell Sci 107:1687-1695.

Tan AM, Colletti M, Rorai AT, Skene JH, Levine JM (2006) Antibodies against the NG2 proteoglycan promote the regeneration of sensory axons within the dorsal columns of the spinal cord. J Neurosci 26:4729-4739.

Tan AM, Petruska JC, Mendell LM, Levine JM (2007) Sensory afferents regenerated into dorsal columns after spinal cord injury remain in a chronic pathophysiological state. Exp Neurol 206:257-268.

Tsirka SE, Gualandris A, Amaral DG, Strickland S (1995) Excitotoxininduced neuronal degeneration and seizure are mediated by tissue plasminogen activator. Nature 377:340-344.

Tsirka SE, Rogove AD, Bugge TH, Degen JL, Strickland S (1997) An extracellular proteolytic cascade promotes neuronal degeneration in the mouse hippocampus. J Neurosci 17:543-552.

Ughrin YM, Chen ZJ, Levine JM (2003) Multiple regions of the NG2 proteoglycan inhibit neurite growth and induce growth cone collapse. J Neurosci 23:175-186.

Vassalli JD, Belin D (1987) Amiloride selectively inhibits the urokinase-type plasminogen activator. FEBS Lett 214:187-191.

Veeravalli KK, Dasari VR, Tsung AJ, Dinh DH, Gujrati M, Fassett D, Rao JS (2009) Stem cells downregulate the elevated levels of tissue plasminogen activator in rats after spinal cord injury. Neurochem Res 34:1183-1194.

Wang H, Katagiri Y, McCann TE, Unsworth E, Goldsmith P, Yu ZX, Tan F, Santiago L, Mills EM, Wang Y, Symes AJ, Geller HM (2008) Chondroitin-4-sulfation negatively regulates axonal guidance and growth. J Cell Sci 121:3083-3091.

Wu YP, Siao CJ, Lu W, Sung TC, Frohman MA, Milev P, Bugge TH, Degen JL, Levine JM, Margolis RU, Tsirka SE (2000) The tissue plasminogen activator (tPA)/plasmin extracellular proteolytic system regulates seizureinduced hippocampal mossy fiber outgrowth through a proteoglycan substrate. J Cell Biol 148:1295-1304.

Yick LW, Wu W, So KF, Yip HK, Shum DK (2000) Chondroitinase ABC promotes axonal regeneration of Clarke's neurons after spinal cord injury. Neuroreport 11:1063-1067. 